CUBO A Mathematical Journal Vol.13, $N^{0}$ 03, (17-48). October 2011

\title{
Applications and Lipschitz results of Approximation by Smooth Picard and Gauss-Weierstrass Type Singular Integrals
}

\author{
RAZvAN A. MEZEI \\ The University of Memphis, \\ Department of Mathematical Sciences, \\ Memphis, TN 38152, U.S.A. \\ email: rmezei@memphis.edu
}

\begin{abstract}
We continue our studies in higher order uniform convergence with rates and in $\mathrm{L}_{p}$ convergence with rates. Namely, in this article we establish some Lipschitz type results for the smooth Picard type singular integral operators and for the smooth GaussWeierstrass type singular integral operators.

\section{RESUMEN}

Continuamos nuestros estudios sobre convergencia uniforme de orden superior con radios y sobre convergencia $L_{p}$ con radios. Concretamente, en este artículo establecemos algunos resultados de tipo Lipschitz para operadores integrales suves del tipo Picard singulares y para operadores integrales singulares de tipo Gauss-Weierstrass.
\end{abstract}

Keywords: Smooth Picard Type singular integral, Smooth Gauss-Weierstrass Type singular integral, modulus of smoothness, rate of convergence, Lp convergence, Higher Order Uniform Convergence with Rates, sharp inequality, Lipschitz functions.

Mathematics Subject Classification: 26A15, 26D15, 41A17, 41A35, 41A60, 41A80. 


\section{Introduction}

We are motivated by [1], [2], [3] and [4].

We denote by $L_{p}, 1 \leq p<\infty$, the classes of functions $f(x)$, integrable in $-\infty<x<\infty$ with the norm

$$
\|f\|_{p}=\left[\int_{-\infty}^{\infty}|f(u)|^{p} d u\right]^{\frac{1}{p}}
$$

The Picard singular integral $P_{\xi}(f ; x)$ corresponding to the function $f(x)$, is defined as follows

$$
P_{\xi}(f ; x)=\frac{1}{2 \xi} \int_{-\infty}^{\infty} f(x+y) e^{-|y| / \xi} d y, \text { for all } x \in \mathbb{R}, \xi>0 .
$$

The Gauss Weierstrass singular integral $W_{\xi}(f ; x)$ corresponding to the function $f(x)$, is defined as follows

$$
W_{\xi}(f ; x)=\frac{1}{\sqrt{\pi \xi}} \int_{-\infty}^{\infty} f(x+y) e^{-y^{2} / \xi} d y, \text { for all } x \in \mathbb{R}, \xi>0 .
$$

\section{Convergence with Rates of Smooth Picard Singular In- tegral Operators}

In the next we deal with the following smooth Picard singular integral operators $\mathrm{P}_{\mathrm{r}, \xi}(f ; x)$ defined as follows.

For $r \in \mathbb{N}$ and $n \in \mathbb{Z}_{+}$we set

$$
\alpha_{j}= \begin{cases}(-1)^{r-j}\left(\begin{array}{l}
r \\
j
\end{array}\right) j^{-n}, & j=1, \ldots, r, \\
1-\sum_{j=1}^{r}(-1)^{r-j}\left(\begin{array}{l}
r \\
j
\end{array}\right) j^{-n}, & j=0,\end{cases}
$$

that is $\sum_{j=0}^{r} \alpha_{j}=1$. Let $f: \mathbb{R} \rightarrow \mathbb{R}$ be Lebesgue measurable, we define for $x \in \mathbb{R}, \xi>0$ the Lebesgue integral

$$
P_{r, \xi}(f ; x):=\frac{1}{2 \xi} \int_{-\infty}^{\infty}\left(\sum_{j=0}^{r} \alpha_{j} f(x+j t)\right) e^{-|t| / \xi} d t .
$$

We assume that $\mathrm{P}_{\mathrm{r}, \xi}(f ; x) \in \mathbb{R}$ for all $x \in \mathbb{R}$.

We mention the useful here formula

$$
\int_{0}^{\infty} t^{k} e^{-t / \xi} d t=\Gamma(k+1) \xi^{k+1}, k>-1 .
$$


We need to introduce

$$
\delta_{k}:=\sum_{j=1}^{r} \alpha_{j} j^{k}, \quad k=1, \ldots, n \in \mathbb{N} .
$$

Denote by $\lfloor\cdot\rfloor$ the integral part.

We give a special related result.

Proposition 1. Let $\mathrm{f}$ be defined as above in this section. It holds that

$$
\left|P_{2, \xi}(f ; x)-f(x)\right| \leq \frac{1}{\xi} \int_{0}^{\infty}\left(\int_{0}^{|t|} \omega_{2}\left(f^{\prime}, w\right) d w\right) e^{-t / \xi} d t
$$

Proof. In Theorem 1 of [1] we use $n=1, r=2$.

We also present the Lipschitz type result corresponding to the Theorem 1 of [1].

Theorem 2. Let $f$ be defined as above in this section, with $n \in \mathbb{N}$. Furthermore we assume the following Lipschitz condition: $\omega_{\mathrm{r}}\left(\mathrm{f}^{(\mathrm{n})}, \delta\right) \leq \mathrm{K} \delta^{\mathrm{r}-1+\gamma}, \mathrm{K}>0,0<\gamma \leq 1$, for any $\delta>0$. Then it holds that

$$
\left|P_{r, \xi}(f ; x)-f(x)-\sum_{m=1}^{\left\lfloor\frac{n}{2}\right\rfloor} f^{(2 m)}(x) \delta_{2 m} \xi^{2 m}\right| \leq K \Gamma(\gamma+r) \xi^{n+r+\gamma-1} .
$$

In L.H.S.(2.6) the sum collapses when $\mathrm{n}=1$.

Proof. As in the proof of Theorem 1, of [1], we get again that

$$
P_{r, \xi}(f ; x)-f(x)=\sum_{k=1}^{n} \frac{f^{(k)}(x)}{k !} \delta_{k} \frac{1}{2 \xi}\left(\int_{-\infty}^{\infty} t^{k} e^{-|t| / \xi} d t\right)+\mathcal{R}_{n}^{*},
$$

where

$$
\mathcal{R}_{n}^{*}:=\frac{1}{2 \xi} \int_{-\infty}^{\infty} \mathcal{R}_{n}(0, t) e^{-|t| / \xi} d t
$$

with

$$
\mathcal{R}_{\mathrm{n}}(0, \mathrm{t}):=\int_{0}^{\mathrm{t}} \frac{(\mathrm{t}-w)^{\mathrm{n}-1}}{(\mathrm{n}-1) !} \tau(w) \mathrm{d} w
$$

and

$$
\tau(w):=\sum_{j=0}^{r} \alpha_{j} j^{n} f^{(n)}(x+j w)-\delta_{n} f^{(n)}(x)
$$

Also we get

$$
\left|\mathcal{R}_{n}(0, t)\right| \leq \int_{0}^{|t|} \frac{(|t|-w)^{n-1}}{(n-1) !} \omega_{r}\left(f^{(n)}, w\right) d w .
$$


Using the Lipschitz type condition we obtain

$$
\begin{aligned}
\left|\mathcal{R}_{n}(0, t)\right| & \leq \int_{0}^{|t|} \frac{(|t|-w)^{n-1}}{(n-1) !} K w^{r-1+\gamma} \mathrm{d} w \\
& =\frac{K|t|^{n+r+\gamma-2}}{(n-1) !} \int_{0}^{|t|}\left(1-\frac{w}{|t|}\right)^{n-1}\left(\frac{w}{|t|}\right)^{r-1+\gamma} d w \\
& =\frac{K|t|^{n+r+\gamma-1}}{(n-1) !} \int_{0}^{1}(1-y)^{n-1} y^{r-1+\gamma} d y \\
& =\frac{K|t|^{n+r+\gamma-1} \Gamma(\gamma+r)}{\Gamma(n+\gamma+r)} .
\end{aligned}
$$

Then, by (2.3), we obtain

$$
\begin{aligned}
\left|\mathcal{R}_{\mathrm{n}}^{*}\right| & \leq \frac{1}{2 \xi} \int_{-\infty}^{\infty} \frac{\mathrm{K}|\mathrm{t}|^{\mathrm{n}+\mathrm{r}+\gamma-1} \Gamma(\gamma+\mathrm{r})}{\Gamma(\mathrm{n}+\gamma+\mathrm{r})} e^{-|\mathrm{t}| / \xi} \mathrm{dt} \\
& =\frac{\mathrm{K}}{2 \xi} \frac{\Gamma(\gamma+\mathrm{r})}{\Gamma(\mathrm{n}+\gamma+\mathrm{r})} \int_{-\infty}^{\infty}|\mathrm{t}|^{\mathrm{n}+\mathrm{r}+\gamma-1} e^{-|\mathrm{t}| / \xi} d t \\
& =\frac{\mathrm{K}}{\xi} \frac{\Gamma(\gamma+r)}{\Gamma(n+\gamma+r)} \int_{0}^{\infty} \mathrm{t}^{\mathrm{n}+\mathrm{r}+\gamma-1} e^{-\mathrm{t} / \xi} \mathrm{dt} \\
& \stackrel{(2.3)}{=} \mathrm{K} \Gamma(\gamma+r) \xi^{n+r+\gamma-1} .
\end{aligned}
$$

We also notice that

$$
\begin{aligned}
P_{r, \xi}(f ; x)-f(x)-\sum_{k=1}^{n} \frac{f^{(k)}(x)}{k !} \delta_{k} \frac{1}{2 \xi}\left(\int_{-\infty}^{\infty} t^{k} e^{-|t| / \xi} d t\right) & = \\
P_{r, \xi}(f ; x)-f(x)-\sum_{m=1}^{\left\lfloor\frac{n}{2}\right\rfloor} f^{(2 m)}(x) \delta_{2 m} \xi^{2 m} & =\mathcal{R}_{n}^{*} .
\end{aligned}
$$

By (2.12) and (2.13) we complete the proof of the theorem.

Corollary 3. Let $f$ be defined as above in this section. Furthermore we assume the following Lipschitz condition $\omega_{2}\left(f^{\prime}, \delta\right) \leq K \delta^{1+\gamma}, K>0,0<\gamma \leq 1$, for any $\delta>0$. Then

$$
\left|P_{2, \xi}(f ; x)-f(x)\right| \leq K \Gamma(\gamma+2) \xi^{2+\gamma}
$$

Proof. In Theorem 2 we use $n=1, r=2$.

For the case $n=0$ we have

Theorem 4. Let $\mathrm{f}$ be defined as above in this section, with $\mathrm{n}=0$. Furthermore we assume the following Lipschitz condition: $\omega_{\mathrm{r}}(\mathrm{f}, \delta) \leq \mathrm{K} \delta^{\mathrm{r}-1+\gamma}, \mathrm{K}>0,0<\gamma \leq 1$, for any $\delta>0$. It holds that

$$
\left|P_{r, \xi}(f ; x)-f(x)\right| \leq K \Gamma(r+\gamma) \xi^{r+\gamma-1} .
$$


Proof. As in the proof of Corollary 1, of [1], with $n=0$, using the Lipschitz type condition, we get that

$$
\begin{aligned}
\left|P_{r, \xi}(f ; x)-f(x)\right| & \leq \frac{1}{\xi} \int_{0}^{\infty} \omega_{r}(f, t) e^{-t / \xi} d t \\
& \leq \frac{1}{\xi} \int_{0}^{\infty} K t^{r-1+\gamma} e^{-t / \xi} d t \\
& \stackrel{(2.3)}{=} K \Gamma(r+\gamma) \xi^{r+\gamma-1}
\end{aligned}
$$

This completes the proof of Theorem 4 .

Corollary 5. Let $\mathrm{f}$ be defined as above in this section, with $\mathrm{n}=0$. Furthermore we assume the following Lipschitz condition: $\omega_{2}(f, \delta) \leq K \delta^{1+\gamma}, K>0,0<\gamma \leq 1$, for any $\delta>0$. Then

$$
\left|P_{2, \xi}(f ; x)-f(x)\right| \leq K \Gamma(2+\gamma) \xi^{\gamma+1} \text {. }
$$

Proof. In Theorem 4 we use $r=2$.

In the next we consider $f \in \mathbb{C}^{\mathfrak{n}}(\mathbb{R}), n \geq 2$ even and the simple smooth singular operator of symmetric convolution type

$$
P_{\xi}\left(f, x_{0}\right):=\frac{1}{2 \xi} \int_{-\infty}^{\infty} f\left(x_{0}+y\right) e^{-|y| / \xi} d y, \text { for all } x_{0} \in \mathbb{R}, \xi>0 .
$$

That is

$$
P_{\xi}\left(f ; x_{0}\right)=\frac{1}{2 \xi} \int_{0}^{\infty}\left(f\left(x_{0}+y\right)+f\left(x_{0}-y\right)\right) e^{-y / \xi} d y, \text { for all } x_{0} \in \mathbb{R}, \xi>0 .
$$

We assume that $f$ is such that

$$
P_{\xi}\left(f ; x_{0}\right) \in \mathbb{R}, \quad \forall x_{0} \in \mathbb{R}, \forall \xi>0 \text { and } \omega_{2}\left(f^{(\mathfrak{n})}, h\right)<\infty, h>0 .
$$

Note that $\mathrm{P}_{1, \xi}=\mathrm{P}_{\xi}$ and if $\mathrm{P}_{\xi}\left(\mathrm{f} ; \mathrm{x}_{0}\right) \in \mathbb{R}$ then $\mathrm{P}_{\mathrm{r}, \xi}\left(\mathrm{f} ; \mathrm{x}_{0}\right) \in \mathbb{R}$.

Proposition 6. Assume $\omega_{2}(\mathrm{f}, \mathrm{h})<\infty, \mathrm{h}>0$. Furthermore we assume the following Lipschitz condition: $\omega_{2}(f, \delta) \leq K \delta^{1+\gamma}, K>0,0<\gamma \leq 1$, for any $\delta>0$. Then

$$
\left\|P_{\xi}(f)-f\right\|_{\infty} \leq \frac{K \Gamma(2+\gamma)}{2} \xi^{\gamma+1} .
$$

Proof. Using Proposition 1 of [1] we obtain

$$
\begin{aligned}
\left|P_{\xi}\left(f ; x_{0}\right)-f\left(x_{0}\right)\right| & \leq \frac{1}{2 \xi} \int_{0}^{\infty} \omega_{2}(f, y) e^{-y / \xi} d y \\
& \leq \frac{1}{2 \xi} \int_{0}^{\infty} K y^{1+\gamma} e^{-y / \xi} d y \\
& \stackrel{(2.3)}{=} \frac{K \Gamma(2+\gamma)}{2} \xi^{\gamma+1},
\end{aligned}
$$


proving the claim of the proposition.

Let

$$
K_{2}\left(x_{0}\right):=P_{\xi}\left(f ; x_{0}\right)-f\left(x_{0}\right)-\sum_{\rho=1}^{n / 2} f^{(2 \rho)}\left(x_{0}\right) \xi^{2 \rho} .
$$

We give

Theorem 7. Let $\mathrm{f} \in \mathrm{C}^{\mathfrak{n}}(\mathbb{R}), \mathrm{n}$ even, $\mathrm{P}_{\xi}(\mathrm{f})$ real valued. Furthermore we assume the following Lipschitz condition: $\omega_{2}\left(f^{(n)}, \delta\right) \leq K \delta^{1+\gamma}, K>0,0<\gamma \leq 1$, for any $\delta>0$. Then

$$
\left|K_{2}\left(x_{0}\right)\right| \leq \frac{K \Gamma(n+\gamma+2)}{2 n !} \xi^{n+\gamma+1} .
$$

Proof. Using Theorem 6 of [1] we obtain

$$
\begin{aligned}
\left|K_{2}\left(x_{0}\right)\right| & \leq \frac{1}{2 \xi n !} \int_{0}^{\infty} \omega_{2}\left(f^{(n)}, y\right) y^{n} e^{-y / \xi} d y \\
& \leq \frac{1}{2 \xi n !} \int_{0}^{\infty} K y^{1+\gamma} y^{n} e^{-y / \xi} d y \\
& \stackrel{(2.3)}{=} \frac{K \Gamma(n+\gamma+2)}{2 n !} \xi^{n+\gamma+1},
\end{aligned}
$$

proving the claim of the theorem.

In particular we have

Corollary 8. Let $\mathrm{f} \in \mathrm{C}^{4}(\mathbb{R})$ such that $\mathrm{P}_{\xi}(\mathrm{f})$ is real valued. Furthermore we assume the following Lipschitz condition: $\omega_{2}\left(\mathrm{f}^{(4)}, \delta\right) \leq \mathrm{K} \delta^{1+\gamma}, \mathrm{K}>0,0<\gamma \leq 1$, for any $\delta>0$. Then

$$
\left|\mathrm{K}_{2}\left(\mathrm{x}_{0}\right)\right| \leq \frac{\mathrm{K} \Gamma(\gamma+6)}{48} \xi^{\gamma+5} .
$$

Proof. In Theorem 7 we use $n=4$.

We also give

Corollary 9. Let $\mathrm{f} \in \mathrm{C}^{2}(\mathbb{R})$, such that

$$
\omega_{2}\left(f^{\prime \prime},|y|\right) \leq 2 A|y|^{\gamma}, \quad 0<\gamma \leq 2, \quad A>0 .
$$

Then for $\mathrm{x}_{0} \in \mathbb{R}$ we have

$$
\left|P_{\xi}\left(f ; x_{0}\right)-f\left(x_{0}\right)-f^{\prime \prime}\left(x_{0}\right) \xi^{2}\right| \leq \Gamma(\alpha+1) A \xi^{\gamma+2} .
$$


Inequality (2.16) is sharp, namely it is attained at $x_{0}=0$ by

$$
f_{*}(y)=\frac{A|y|^{\gamma+2}}{(\gamma+1)(\gamma+2)} .
$$

Proof. In Theorem 7 of [1] we use $n=2$.

We also give

Corollary 10. Assume that $\omega_{2}(f, \xi)<\infty$ and $n=0$. Then

$$
\left\|P_{2, \xi}(f)-f\right\|_{\infty} \leq 5 \omega_{2}(f, \xi),
$$

and as $\xi \rightarrow 0$,

$$
\mathrm{P}_{2, \xi} \stackrel{\text { u }}{\rightarrow} \text { I with rates. }
$$

Proof. By formula (37) of [1] with $r=2$.

Next let

$$
K_{1}:=\left\|P_{r, \xi}(f ; x)-f(x)-\sum_{m=1}^{\lfloor n / 2\rfloor}\left[f^{(2 m)}(x) \delta_{2 m} \xi^{2 m}\right]\right\|_{\infty, x} .
$$

We present

Corollary 11. Assuming $f \in C^{2}(\mathbb{R})$ and $\omega_{2}\left(f^{\prime \prime}, \xi\right)<\infty, \xi>0$ we have

$$
\begin{aligned}
K_{1} & =\left\|P_{2, \xi}(f ; x)-f(x)-f^{\prime \prime}(x) \delta_{2} \xi^{2}\right\|_{\infty, x} \\
& \leq \frac{21}{4} \xi^{2} \omega_{2}\left(f^{\prime \prime}, \xi\right) .
\end{aligned}
$$

That is as $\xi \rightarrow 0$ we get $\mathrm{P}_{2, \xi} \rightarrow \mathrm{I}$, pointwise with rates, given that $\left\|\mathrm{f}^{\prime \prime}\right\|_{\infty}<\infty$.

Proof. In Theorem 11 of [1] we use $r=n=2$.

We also present

Corollary 12. Assuming $f \in \mathrm{C}^{2}(\mathbb{R})$ and $\omega_{2}\left(\mathrm{f}^{\prime \prime}, \xi\right)<\infty, \xi>0$ we have

$$
\begin{aligned}
\left\|K_{2}(x)\right\|_{\infty, x} & =\left\|P_{\xi}\left(f ; x_{0}\right)-f\left(x_{0}\right)-f^{\prime \prime}\left(x_{0}\right) \xi^{2}\right\|_{\infty, x} \\
& \leq \frac{21}{8} \xi^{2} \omega_{2}\left(f^{\prime \prime}, \xi\right) .
\end{aligned}
$$

That is as $\xi \rightarrow 0$ we get $\mathrm{P}_{\xi} \rightarrow \mathrm{I}$, pointwise with rates, given that $\left\|\mathrm{f}^{\prime \prime}\right\|_{\infty}<\infty$.

Proof. In Theorem 12 of [1] we use $n=2$. 


\section{3. $\mathbf{L}_{\mathrm{p}}$ Convergence with Rates of Smooth Picard Singular Integral Operators}

For $r \in \mathbb{N}$ and $n \in \mathbb{Z}_{+}$we let $\alpha_{j}$ as in $(2.1)$.

Let $f \in C^{n}(\mathbb{R})$ and $f^{(n)} \in L_{p}(\mathbb{R}), 1 \leq p<\infty$, we define for $x \in \mathbb{R}, \xi>0$ the Lebesgue integral $\mathrm{P}_{\mathrm{r}, \xi}(\mathrm{f} ; \mathrm{x})$ as in $(2.2)$.

We need the rth $\mathrm{L}_{p}$-modulus of smoothness

$$
\omega_{r}\left(f^{(n)}, h\right)_{p}:=\sup _{|t| \leq h}\left\|\Delta_{t}^{r} f^{(n)}(x)\right\|_{p, x}, \quad h>0
$$

where

$$
\Delta_{t}^{r} f^{(n)}(x):=\sum_{j=0}^{r}(-1)^{r-j}\left(\begin{array}{l}
r \\
j
\end{array}\right) f^{(n)}(x+j t),
$$

Here we have that $\omega_{r}\left(f^{(n)}, h\right)_{p}<\infty, h>0$.

We need to introduce $\delta_{k}$ 's as in (2.4).

We define

$$
\Delta(x):=P_{r, \xi}(f ; x)-f(x)-\sum_{m=1}^{\lfloor n / 2\rfloor} f^{(2 m)}(x) \delta_{2 m} \xi^{2 m}
$$

We have the following results.

Corollary 13. Let $\mathrm{n} \in \mathbb{N}$ and the rest as above in this section. Then

$$
\|\Delta(x)\|_{2} \leq \frac{\sqrt{2 \tau} \xi^{n}}{\sqrt{(2 r+1)(4 n-2)}(n-1) !} \omega_{r}\left(f^{(n)}, \xi\right)_{2},
$$

where

$$
0<\tau:=\left[\int_{0}^{\infty}(1+u)^{2 r+1} u^{2 n-1} e^{-u} d u-(2 n-1) !\right]<\infty .
$$

Hence as $\xi \rightarrow 0$ we obtain $\|\Delta(\mathrm{x})\|_{2} \rightarrow 0$.

If additionally $f^{(2 m)} \in L_{2}(\mathbb{R}), m=1,2, \ldots,\left\lfloor\frac{n}{2}\right\rfloor$ then $\left\|P_{r, \xi}(f)-f\right\|_{2} \rightarrow 0$, as $\xi \rightarrow 0$.

Proof. In Theorem 1 of [2], we place $p=q=2$.

Corollary 14. Let $\mathrm{f}$ be as above in this section. In particular, for $\mathrm{n}=1$, we have

$$
\left\|P_{r, \xi}(f ; \cdot)-f\right\|_{2} \leq \frac{\sqrt{\tau} \xi}{\sqrt{(2 r+1)}} \omega_{r}\left(f^{\prime}, \xi\right)_{2},
$$


where

$$
0<\tau:=\left[\int_{0}^{\infty}(1+u)^{2 r+1} u e^{-u} d u-1\right]<\infty .
$$

Hence as $\xi \rightarrow 0$ we obtain $\left\|\mathrm{P}_{\mathrm{r}, \xi}(\mathrm{f} ; \cdot)-\mathrm{f}\right\|_{2} \rightarrow 0$.

Proof. In Theorem 1 of [2], we place $p=q=2, n=1$.

Corollary 15. Let $\mathrm{f}$ be as above in this section and $\mathrm{n}=2$. Then

$$
\left\|P_{r, \xi}(f ; x)-f(x)-f^{\prime \prime}(x) \delta_{2} \xi^{2}\right\|_{2} \leq \frac{\sqrt{2 \tau} \xi^{2}}{\sqrt{6(2 r+1)}} \omega_{r}\left(f^{\prime \prime}, \xi\right)_{2},
$$

where

$$
0<\tau:=\left[\int_{0}^{\infty}(1+u)^{2 r+1} u^{3} e^{-u} d u-6\right]<\infty .
$$

Hence as $\xi \rightarrow 0$ we obtain $\|\Delta(\mathrm{x})\|_{2} \rightarrow 0$.

If additionally $f^{\prime \prime} \in L_{2}(\mathbb{R})$, then $\left\|P_{r, \xi}(f)-f\right\|_{2} \rightarrow 0$, as $\xi \rightarrow 0$.

Proof. In Theorem 1 of [2], we place $p=q=n=2$.

Next we present the Lipschitz type result corresponding to Theorem 1 of [2].

Theorem 16. Let $\mathrm{p}, \mathrm{q}>1$ such that $\frac{1}{\mathrm{p}}+\frac{1}{\mathrm{q}}=1, \mathrm{n} \in \mathbb{N}$, and the rest as above in this section. Furthermore we assume the following Lipschitz condition: $\omega_{\mathrm{r}}\left(\mathrm{f}^{(\mathfrak{n})}, \delta\right)_{\mathrm{p}} \leq \mathrm{K} \delta^{\mathrm{r}-1+\gamma}, \mathrm{K}>0$, $0<\gamma \leq 1$, for any $\delta>0$. Then

$$
\|\Delta(x)\|_{p} \leq \frac{(\Gamma(p(r-1+\gamma+n)+1))^{\frac{1}{p}} 2^{(r+\gamma+n)} K}{\left[(n-1) ! q^{\frac{1}{q}} p^{r-\frac{1}{q}+\gamma+n}(q(n-1)+1)^{\frac{1}{q}}(p(r-1+\gamma)+1)^{\frac{1}{p}}\right]} \xi^{(r-1+\gamma+n)} .
$$

Hence as $\xi \rightarrow 0$ we obtain $\|\Delta(\mathrm{x})\|_{\mathrm{p}} \rightarrow 0$.

If additionally $f^{(2 m)} \in L_{p}(\mathbb{R}), m=1,2, \ldots,\left\lfloor\frac{n}{2}\right\rfloor$ then $\left\|P_{r, \xi}(f)-f\right\|_{p} \rightarrow 0$, as $\xi \rightarrow 0$.

Proof. As in the proof of Theorem 1, [2], we get again

$$
\begin{aligned}
I \quad & =\int_{-\infty}^{\infty}|\Delta(x)|^{p} d x \\
& \leq c_{1}\left(\int_{-\infty}^{\infty}\left(\left(\int_{0}^{|t|} \omega_{r}\left(f^{(\mathfrak{n})}, w\right)_{p}^{p} d w\right)|t|^{n p-1} e^{-|p t| / 2 \xi}\right) d t\right),
\end{aligned}
$$

where

$$
c_{1}:=\frac{2^{p-2}}{\xi q^{p-1}((n-1) !)^{p}(q(n-1)+1)^{p / q}} .
$$


Using the Lipschitz condition, we obtain

$$
\begin{aligned}
I & \leq c_{1}\left(\int_{-\infty}^{\infty}\left(\int_{0}^{|t|}\left(K w^{r-1+\gamma}\right)^{p} d w\right)|t|^{n p-1} e^{-p|t| / 2 \xi} d t\right) \\
& =\frac{c_{1} K^{p}}{(p(r-1+\gamma)+1)}\left(\int_{-\infty}^{\infty}|t|^{p(r-1+\gamma+n)} e^{-p|t| / 2 \xi} d t\right) \\
& =\frac{2 c_{1} K^{p}}{(p(r-1+\gamma)+1)}\left(\int_{0}^{\infty} t^{p(r-1+\gamma+n)} e^{-p t / 2 \xi} d t\right) \\
& =\frac{2 c_{1} K^{p}}{(p(r-1+\gamma)+1)}\left(\frac{2}{p}\right)^{p(r-1+\gamma+n)+1}\left(\int_{0}^{\infty} z^{p(r-1+\gamma+n)} e^{-z / \xi} d z\right) \\
& \stackrel{2.3)}{=} \frac{2 c_{1} K^{p} \Gamma(p(r-1+\gamma+n)+1)}{(p(r-1+\gamma)+1)}\left(\frac{2}{p}\right)^{p(r-1+\gamma+n)+1} \xi^{p(r-1+\gamma+n)+1} .
\end{aligned}
$$

Thus we obtain

$$
I \leq \frac{\Gamma(p(r-1+\gamma+n)+1)}{q^{p-1}((n-1) !)^{p}(q(n-1)+1)^{p / q} p^{p(r-1+\gamma+n)+1}} \frac{2^{p(r+\gamma+n)} K^{p}}{(p(r-1+\gamma)+1)} \xi^{p(r-1+\gamma+n)} .
$$

That is finishing the proof of the theorem.

In particular we have

Corollary 17. Let $\mathrm{f}$ such that the following Lipschitz condition holds: $\omega_{7}\left(\mathrm{f}^{(4)}, \delta\right)_{2} \leq \mathrm{K} \delta^{6+\gamma}$, $\mathrm{K}>0,0<\gamma \leq 1$, for any $\delta>0$, and the rest as above in this section. Then

$$
\|\Delta(x)\|_{2} \leq \frac{K}{6} \sqrt{\frac{(\Gamma(2 \gamma+21))}{7(2 \gamma+13)}} \xi^{(\gamma+10)} .
$$

Hence as $\xi \rightarrow 0$ we obtain $\|\Delta(\mathrm{x})\|_{2} \rightarrow 0$.

If additionally $f^{(2 m)} \in L_{2}(\mathbb{R}), m=1,2$, then $\left\|P_{7, \xi}(f)-f\right\|_{2} \rightarrow 0$, as $\xi \rightarrow 0$.

Proof. In Theorem 16 we place $p=q=2, n=4$, and $r=7$.

The counterpart of Theorem 16 follows, case of $p=1$.

Theorem 18. Let $f \in \mathrm{C}^{\mathrm{n}}(\mathbb{R})$ and $\mathrm{f}^{(\mathrm{n})} \in \mathrm{L}_{1}(\mathbb{R}), \mathrm{n} \in \mathbb{N}$. Furthermore we assume the following Lipschitz condition: $\omega_{\mathrm{r}}\left(\mathrm{f}^{(\mathrm{n})}, \delta\right)_{1} \leq \mathrm{K} \delta^{\mathrm{r}-1+\gamma}, \mathrm{K}>0,0<\gamma \leq 1$, for any $\delta>0$. Then

$$
\|\Delta(x)\|_{1} \leq \frac{K}{(n-1) !(r+\gamma)} \Gamma(r+\gamma+n) \xi^{r+\gamma+n-1} .
$$

Hence as $\xi \rightarrow 0$ we obtain $\|\Delta(x)\|_{1} \rightarrow 0$. 
If additionally $f^{(2 m)} \in L_{1}(\mathbb{R}), m=1,2, \ldots,\left\lfloor\frac{n}{2}\right\rfloor$ then $\left\|P_{r, \xi}(f)-f\right\|_{1} \rightarrow 0$, as $\xi \rightarrow 0$.

Proof. As in the proof of Theorem 2 of [2] we get

$$
\|\Delta(x)\|_{1} \leq \frac{1}{2 \xi(n-1) !}\left(\int_{-\infty}^{\infty}\left(\int_{0}^{|t|} \omega_{r}\left(f^{(n)}, w\right)_{1} d w\right)|t|^{n-1} e^{-|t| / \xi} d t\right) .
$$

Consequently we have

$$
\begin{aligned}
\|\Delta(x)\|_{1} & \leq \frac{1}{2 \xi(n-1) !}\left(\int_{-\infty}^{\infty}\left(\int_{0}^{|t|} K w^{r-1+\gamma} \mathrm{d} w\right)|t|^{n-1} e^{-|t| / \xi} d t\right) \\
& =\frac{K}{2 \xi(n-1) !}\left(\int_{-\infty}^{\infty}\left(\frac{|t|^{r+\gamma}}{r+\gamma}\right)|t|^{n-1} e^{-|t| / \xi} d t\right) \\
& =\frac{K}{2 \xi(n-1) !(r+\gamma)}\left(\int_{-\infty}^{\infty}|t|^{r+\gamma+n-1} e^{-|t| / \xi} d t\right) \\
& =\frac{K}{\xi(n-1) !(r+\gamma)}\left(\int_{0}^{\infty} t^{r+\gamma+n-1} e^{-t / \xi} d t\right) \\
& \stackrel{K}{=} \frac{K}{(n-1) !(r+\gamma)} \Gamma(r+\gamma+n) \xi^{r+\gamma+n-1},
\end{aligned}
$$

proving (3.16).

Corollary 19. Let $f \in \mathrm{C}^{2}(\mathbb{R})$ and $\mathrm{f}^{\prime \prime} \in \mathrm{L}_{1}(\mathbb{R})$. Furthermore we assume the following Lipschitz condition: $\omega_{2}\left(f^{\prime \prime}, \delta\right)_{1} \leq K \delta^{1+\gamma}, K>0,0<\gamma \leq 1$, for any $\delta>0$. Then

$$
\|\Delta(x)\|_{1} \leq \frac{K}{(2+\gamma)} \Gamma(4+\gamma) \xi^{\gamma+3} .
$$

Hence as $\xi \rightarrow 0$ we obtain $\|\Delta(\mathrm{x})\|_{1} \rightarrow 0$.

If additionally $f^{\prime \prime} \in \mathrm{L}_{1}(\mathbb{R})$, then $\left\|\mathrm{P}_{2, \xi}(\mathrm{f})-\mathrm{f}\right\|_{1} \rightarrow 0$, as $\xi \rightarrow 0$.

Proof. In Theorem 18 we place $n=r=2$.

Next, when $\mathfrak{n}=0$ we get

Proposition 20. Let $\mathrm{r} \in \mathbb{N}$ and the rest as above. Then

$$
\left\|P_{r, \xi}(f)-f\right\|_{2} \leq \theta^{1 / 2} \omega_{r}(f, \xi)_{2},
$$

where

$$
0<\theta:=\int_{0}^{\infty}(1+x)^{2 r} e^{-x} \mathrm{~d} x<\infty
$$

Hence as $\xi \rightarrow 0$ we obtain $\mathrm{P}_{\mathrm{r}, \xi} \rightarrow$ unit operator I in the $\mathrm{L}_{2}$ norm. 
Proof. In the proof of Proposition 1 of [2] we use $p=q=2$.

We continue with

Proposition 21. Let $\mathrm{p}, \mathrm{q}>1$ such that $\frac{1}{\mathrm{p}}+\frac{1}{\mathrm{q}}=1$ and the rest as above. Furthermore we assume the following Lipschitz condition: $\omega_{\mathrm{r}}(\mathrm{f}, \delta)_{\mathrm{p}} \leq \mathrm{K} \delta^{\mathrm{r}-1+\gamma}, \mathrm{K}>0,0<\gamma \leq 1$, for any $\delta>0$. Then

$$
\left\|P_{r, \xi}(f)-f\right\|_{p} \leq \sqrt[p]{\Gamma(p(r-1+\gamma)+1)} \frac{K}{q^{1 / q}} \frac{2^{(r+\gamma)} \xi^{(r+\gamma-1)}}{p^{\left(r-1+\gamma+\frac{1}{p}\right)}} .
$$

Hence as $\xi \rightarrow 0$ we obtain $\mathrm{P}_{\mathrm{r}, \xi} \rightarrow$ unit operator $\mathrm{I}$ in the $\mathrm{L}_{\mathrm{p}}$ norm, $\mathrm{p}>1$.

Proof. As in the proof of Proposition 1 of [2] we find

$$
\begin{gathered}
\int_{-\infty}^{\infty}\left|P_{r, \xi}(f ; x)-f(x)\right|^{p} d x \\
\leq \frac{1}{2^{p-1} \xi^{p}}\left(\frac{4 \xi}{q}\right)^{p / q}\left(\int_{0}^{\infty} \omega_{r}(f, t)_{p}^{p} e^{-p t /(2 \xi)} d t\right) \\
\leq \frac{1}{2^{p-1} \xi^{p}}\left(\frac{4 \xi}{q}\right)^{p / q}\left(\int_{0}^{\infty}\left(K t^{r-1+\gamma}\right)^{p} e^{-p t /(2 \xi)} d t\right) \\
\stackrel{(2.3)}{=} \frac{K^{p}}{q^{p-1}} \frac{\Gamma(p(r-1+\gamma)+1) 2^{p(r+\gamma)} \xi^{(r-1+\gamma) p}}{p^{(p(r+\gamma-1)+1)}} .
\end{gathered}
$$

We have established the claim of the proposition.

Corollary 22. Let $f$ such that the following Lipschitz condition holds: $\omega_{4}(\mathrm{f}, \delta)_{2} \leq \mathrm{K} \delta^{3+\gamma}$, $\mathrm{K}>0,0<\gamma \leq 1$, for any $\delta>0$, and the rest as above in this section. Then

$$
\left\|P_{4, \xi}(f)-f\right\|_{2} \leq \sqrt{\Gamma(2 \gamma+7)} K \xi^{(3+\gamma)} .
$$

Hence as $\xi \rightarrow 0$ we obtain $\mathrm{P}_{4, \xi} \rightarrow$ unit operator $\mathrm{I}$ in the $\mathrm{L}_{2}$ norm.

Proof. In Proposition 21 we place $p=q=2$ and $r=4$.

In general, for the $L_{1}$ case, $n=0$ we have

Proposition 23. It holds

$$
\left\|P_{2, \xi} f-f\right\|_{1} \leq 5 \omega_{2}(f, \xi)_{1} .
$$

Hence as $\xi \rightarrow 0$ we get $\mathrm{P}_{2, \xi} \rightarrow \mathrm{I}$ in the $\mathrm{L}_{1}$ norm.

Proof. In the proof of Proposition 2 of [2] we use $r=2$.

Proposition 24. We assume the following Lipschitz condition: $\omega_{\mathrm{r}}(\mathrm{f}, \delta)_{1} \leq \mathrm{K} \delta^{\mathrm{r}-1+\gamma}, \mathrm{K}>0$, $0<\gamma \leq 1$, for any $\delta>0$. Then

$$
\left\|P_{r, \xi} f-f\right\|_{1} \leq K \Gamma(r+\gamma) \xi^{r-1+\gamma}
$$


Hence as $\xi \rightarrow 0$ we get $\mathrm{P}_{\mathrm{r}, \xi} \rightarrow \mathrm{I}$ in the $\mathrm{L}_{1}$ norm.

Proof. As in the proof of Proposition 2 of [2] we get

$$
\begin{aligned}
\int_{-\infty}^{\infty}\left|P_{r, \xi}(f ; x)-f(x)\right| d x & \leq \frac{1}{\xi} \int_{0}^{\infty} \omega_{r}(f, t) e_{1} e^{-t / \xi} d t \\
& \leq \frac{K}{\xi} \int_{0}^{\infty} t^{r-1+\gamma} e^{-t / \xi} d t \\
& =K \Gamma(r+\gamma) \xi^{r-1+\gamma}
\end{aligned}
$$

proving the claim.

Corollary 25. Assume the following Lipschitz condition: $\omega_{2}(f, \delta)_{1} \leq \mathrm{K}^{1+\gamma}, \mathrm{K}>0,0<\gamma \leq$ 1 , for any $\delta>0$. Then

$$
\left\|P_{2, \xi} f-f\right\|_{1} \leq K \Gamma(2+\gamma) \xi^{1+\gamma} .
$$

Hence as $\xi \rightarrow 0$ we get $\mathrm{P}_{2, \xi} \rightarrow \mathrm{I}$ in the $\mathrm{L}_{1}$ norm.

Proof. In Proposition 24 we place $r=2$.

In the next we consider $f \in \mathrm{C}^{\mathrm{n}}(\mathbb{R})$ and $\mathrm{f}^{(\mathrm{n})} \in \mathrm{L}_{\mathrm{p}}(\mathbb{R}), \mathrm{n}=0$ or $\mathrm{n} \geq 2$ even, $1 \leq p<\infty$ and the similar smooth singular operator of symmetric convolution type

$$
P_{\xi}(f ; x)=\frac{1}{2 \xi} \int_{-\infty}^{\infty} f(x+y) e^{-|y| / \xi} d y, \text { for all } x \in \mathbb{R}, \xi>0 .
$$

Denote

$$
K(x):=P_{\xi}(f ; x)-f(x)-\sum_{\rho=1}^{n / 2} f^{(2 \rho)}(x) \xi^{2 \rho} .
$$

We give

Theorem 26. Let $\mathrm{n} \geq 2$ even and the rest as above. Then

$$
\|K(x)\|_{2} \leq\left(\sqrt{\frac{\tilde{\tau}}{20(2 n-1)}}\right) \frac{\xi^{n}}{(n-1) !} \omega_{2}\left(f^{(n)}, \xi\right)_{2},
$$

where

$$
0<\tilde{\tau}=\left(\int_{0}^{\infty}(1+x)^{5} x^{2 n-1} e^{-x} d x-(2 n-1) !\right)<\infty .
$$

Hence as $\xi \rightarrow 0$ we get $\|\mathrm{K}(\mathrm{x})\|_{2} \rightarrow 0$.

If additionally $f^{(2 m)} \in L_{2}(\mathbb{R}), m=1,2, \ldots, \frac{n}{2}$ then $\left\|P_{\xi}(f)-f\right\|_{2} \rightarrow 0$, as $\xi \rightarrow 0$.

Proof. In the proof of Theorem 3 of [2] we use $p=q=2$. 
It follows a Lipschitz type approximation result.

Theorem 27. Let $\mathrm{p}, \mathrm{q}>1$ such that $\frac{1}{\mathrm{p}}+\frac{1}{\mathrm{q}}=1, \mathrm{n} \geq 2$ even and the rest as above. Furthermore we assume the following Lipschitz condition: $\omega_{2}\left(f^{(n)}, \delta\right)_{p} \leq \mathrm{K} \delta^{\gamma+1}, \mathrm{~K}>0,0<\gamma \leq 1$, for any $\delta>0$. Then

$$
\|K(x)\|_{p} \leq\left(\frac{2}{p}\right)^{(\gamma+n+1)} \frac{K[\Gamma(p(\gamma+n+1)+1)]^{1 / p}}{(n-1) ! q^{1 / q} p^{1 / p}(q(n-1)+1)^{1 / q}[p(\gamma+1)+1]^{1 / p}} \xi^{\gamma+n+1} .
$$

Hence as $\xi \rightarrow 0$ we get $\|\mathrm{K}(\mathrm{x})\|_{\mathrm{p}} \rightarrow 0$.

If additionally $f^{(2 m)} \in L_{p}(\mathbb{R}), m=1,2, \ldots, \frac{n}{2}$ then $\left\|P_{\xi}(f)-f\right\|_{p} \rightarrow 0$, as $\xi \rightarrow 0$.

Proof. As in the proof of Theorem 3, of [2] we find

$$
\begin{aligned}
& \int_{-\infty}^{\infty}|K(x)|^{p} d x \leq c_{2}\left(\int_{0}^{\infty}\left(\int_{0}^{y} \omega_{2}\left(f^{(n)}, t\right)_{p}^{p} d t\right) y^{p n-1} e^{-p y /(2 \xi)} d y\right) \\
& \leq K^{p} c_{2}\left(\int_{0}^{\infty}\left(\frac{y^{p(\gamma+1)+1}}{p(\gamma+1)+1}\right) y^{p n-1} e^{-p y /(2 \xi)} d y\right) \\
&=\frac{K^{p} c_{2}}{p(\gamma+1)+1}\left(\frac{2}{p}\right)^{p(\gamma+n+1)+1}\left(\int_{0}^{\infty} z^{p(\gamma+n+1)} e^{-z / \xi} d z\right) \\
& \stackrel{(2.3)}{=} \frac{K^{p} c_{2} \Gamma(p(\gamma+n+1)+1)}{p(\gamma+1)+1}\left(\frac{2}{p}\right)^{p(\gamma+n+1)+1} \xi^{p(\gamma+n+1)+1} .
\end{aligned}
$$

where here we denoted

$$
c_{2}:=\frac{1}{2 \xi q^{p / q}((n-1) !)^{p}(q(n-1)+1)^{p / q}} .
$$

We have established the claim of the theorem.

Corollary 28. Assume the following Lipschitz condition: $\omega_{2}\left(f^{\prime \prime}, \delta\right)_{2} \leq \mathrm{K} \delta^{\gamma+1}, \mathrm{~K}>0,0<$ $\gamma \leq 1$, for any $\delta>0$, and the rest as above in this section. Then

$$
\|\mathrm{K}(x)\|_{2} \leq \sqrt{\frac{\Gamma(2 \gamma+7)}{6 \gamma+9}} \frac{\mathrm{K}}{2} \xi^{\gamma+3}
$$

Hence as $\xi \rightarrow 0$ we get $\|\mathrm{K}(\mathrm{x})\|_{2} \rightarrow 0$.

If additionally $f^{\prime \prime} \in L_{2}(\mathbb{R})$, then $\left\|P_{\xi}(f)-f\right\|_{2} \rightarrow 0$, as $\xi \rightarrow 0$.

Proof. In Theorem 27 we place $p=q=n=2$.

Theorem 29. Let $f \in C^{2}(\mathbb{R})$ and $f^{\prime \prime} \in L_{1}(\mathbb{R})$. Here $K(x)=P_{\xi}(f ; x)-f(x)-f^{\prime \prime}(x) \xi^{2}$. Then

$$
\|K(x)\|_{1} \leq 8 \omega_{2}\left(f^{\prime \prime}, \xi\right)_{1} \xi^{2} .
$$

Hence as $\xi \rightarrow 0$ we obtain $\|\mathrm{K}(\mathrm{x})\|_{1} \rightarrow 0$. 
Also $\left\|P_{\xi}(f)-f\right\|_{1} \rightarrow 0$, as $\xi \rightarrow 0$.

Proof. In the proof of Theorem 4 of [2] we use $n=2$.

The Lipschitz case of $p=1$ follows.

Theorem 30. Let $f \in \mathrm{C}^{\mathfrak{n}}(\mathbb{R})$ and $\mathrm{f}^{(\mathrm{n})} \in \mathrm{L}_{1}(\mathbb{R}), \mathrm{n} \geq 2$ even. Furthermore we assume the following Lipschitz condition: $\omega_{2}\left(\mathrm{f}^{(\mathrm{n})}, \delta\right)_{1} \leq \mathrm{K} \delta^{\gamma+1}, \mathrm{~K}>0,0<\gamma \leq 1$, for any $\delta>0$. Then

$$
\|K(x)\|_{1} \leq \frac{\Gamma(\gamma+n+2) K}{2(n-1) !(\gamma+2)} \xi^{\gamma+n+1} .
$$

Hence as $\xi \rightarrow 0$ we obtain $\|\mathrm{K}(\mathrm{x})\|_{1} \rightarrow 0$.

If additionally $f^{(2 m)} \in L_{1}(\mathbb{R}), m=1,2, \ldots, \frac{n}{2}$ then $\left\|P_{\xi}(f)-f\right\|_{1} \rightarrow 0$, as $\xi \rightarrow 0$.

Proof. As in the proof of Theorem 4 of [2] we have

$$
\begin{aligned}
\|K(x)\|_{1} & \leq \frac{1}{2 \xi}\left(\int_{0}^{\infty}\left(\int_{0}^{y} \omega_{2}\left(f^{(n)}, t\right){ }_{1} d t\right) \frac{y^{n-1}}{(n-1) !} e^{-y / \xi} d y\right) \\
& \leq \frac{1}{2 \xi}\left(\int_{0}^{\infty}\left(\int_{0}^{y} K t^{\gamma+1} d t\right) \frac{y^{n-1}}{(n-1) !} e^{-y / \xi} d y\right) \\
& =\frac{K}{2 \xi(n-1) !(\gamma+2)}\left(\int_{0}^{\infty} y^{\gamma+n+1} e^{-y / \xi} d y\right) \\
& \stackrel{(2.3)}{=} \frac{\Gamma(\gamma+n+2) K}{2(n-1) !(\gamma+2)} \xi^{\gamma+n+1} .
\end{aligned}
$$

We have proved the claim of the theorem.

Corollary 31. Let $f \in C^{6}(\mathbb{R})$ and $f^{(6)} \in L_{1}(\mathbb{R})$. Furthermore we assume the following Lipschitz condition: $\omega_{2}\left(f^{(6)}, \delta\right)_{1} \leq K \delta^{\gamma+1}, K>0,0<\gamma \leq 1$, for any $\delta>0$. Then

$$
\|\mathrm{K}(\mathrm{x})\|_{1} \leq \frac{\Gamma(\gamma+8) \mathrm{K}}{240(\gamma+2)} \xi^{\gamma+7}
$$

Hence as $\xi \rightarrow 0$ we obtain $\|\mathrm{K}(\mathrm{x})\|_{1} \rightarrow 0$.

If additionally $\mathrm{f}^{(2 \mathrm{~m})} \in \mathrm{L}_{1}(\mathbb{R}), \mathrm{m}=1,2,3$ then $\left\|\mathrm{P}_{\xi}(\mathrm{f})-\mathrm{f}\right\|_{1} \rightarrow 0$, as $\xi \rightarrow 0$.

Proof. In Theorem 30 we place $\mathrm{n}=6$.

The case of $n=0$ follows.

Proposition 32. Let $f$ as above in this section. Then

$$
\left\|P_{\xi}(f)-f\right\|_{2} \leq \frac{\sqrt{65}}{2} \omega_{2}(f, \xi)_{2} .
$$

Hence as $\xi \rightarrow 0$ we obtain $\mathrm{P}_{\xi} \rightarrow \mathrm{I}$ in the $\mathrm{L}_{2}$ norm. 
Proof. In the proof of Proposition 3 of [2] we use $p=q=2$.

The related Lipschitz case for $n=0$ comes next.

Proposition 33. Let $\mathrm{p}, \mathrm{q}>1$ such that $\frac{1}{\mathrm{p}}+\frac{1}{\mathrm{q}}=1$ and the rest as above. Furthermore we assume the following Lipschitz condition: $\omega_{2}(\mathrm{f}, \delta)_{\mathrm{p}} \leq \mathrm{K} \delta^{1+\gamma}, \mathrm{K}>0,0<\gamma \leq 1$, for any $\delta>0$. Then

$$
\left\|P_{\xi}(f)-f\right\|_{p} \leq\left(\frac{2}{p}\right)^{1+\gamma} \frac{[\Gamma((1+\gamma) p+1)]^{1 / p} K}{q^{1 / q} p^{1 / p}} \xi^{1+\gamma} .
$$

Hence as $\xi \rightarrow 0$ we obtain $\mathrm{P}_{\xi} \rightarrow \mathrm{I}$ in the $\mathrm{L}_{\mathrm{p}}$ norm, $\mathrm{p}>1$.

Proof. As in the proof of Proposition 3 of [2] we get

$$
\begin{aligned}
& \int_{-\infty}^{\infty}\left|P_{\xi}(f ; x)-f(x)\right|^{p} d x \leq \frac{1}{2 \xi q^{p / q}}\left(\int_{0}^{\infty} \omega_{2}(f, y)_{p}^{p} e^{-p y /(2 \xi)} d y\right) \\
& \leq \frac{K^{p}}{2 \xi q^{p / q}}\left(\int_{0}^{\infty} y^{(1+\gamma) p} e^{-p y /(2 \xi)} d y\right) \\
& \stackrel{(2.3)}{=} \frac{K^{p}}{q^{p / q} p}\left(\frac{2}{p}\right)^{(1+\gamma) p} \Gamma((1+\gamma) p+1) \xi^{(1+\gamma) p} .
\end{aligned}
$$

The proof of the claim is now completed.

A particular example follows

Corollary 34. Let $\mathrm{f}$ as above in this section. Furthermore we assume the following Lipschitz condition: $\omega_{2}(f, \delta)_{2} \leq \mathrm{K} \delta^{1+\gamma}, \mathrm{K}>0,0<\gamma \leq 1$, for any $\delta>0$. Then

$$
\left\|P_{\xi}(f)-f\right\|_{2} \leq \frac{K}{2} \sqrt{\Gamma(3+2 \gamma)} \xi^{1+\gamma} .
$$

Hence as $\xi \rightarrow 0$ we obtain $\mathrm{P}_{\xi} \rightarrow \mathrm{I}$ in the $\mathrm{L}_{2}$ norm.

Proof. In Proposition 33 we place $p=q=2$.

It follows the Lipschitz type result

Proposition 35. Assume the following Lipschitz condition: $\mathrm{\omega}_{2}(\mathrm{f}, \delta)_{1} \leq \mathrm{K} \delta^{\gamma+1}, \mathrm{~K}>0$, $0<\gamma \leq 1$, for any $\delta>0$. It holds,

$$
\left\|P_{\xi} f-f\right\|_{1} \leq \frac{K}{2} \Gamma(\gamma+2) \xi^{\gamma+1} .
$$

Hence as $\xi \rightarrow 0$ we get $\mathrm{P}_{\xi} \rightarrow \mathrm{I}$ in the $\mathrm{L}_{1}$ norm.

Proof. As in the proof of Proposition 4 of [2] we derive

$$
\begin{aligned}
\int_{-\infty}^{\infty}\left|P_{\xi}(f ; x)-f(x)\right| d x & \leq \frac{1}{2 \xi} \int_{0}^{\infty} \omega_{2}(f, y){ }_{1} e^{-y / \xi} d y \\
& \leq \frac{1}{2 \xi} \int_{0}^{\infty} K y^{\gamma+1} e^{-y / \xi} d y \\
\stackrel{(2.3)}{=} & \frac{K}{2} \Gamma(\gamma+2) \xi^{\gamma+1},
\end{aligned}
$$


proving the claim.

\section{Convergence with Rates of Smooth Gauss Weierstrass Singular Integral Operators}

In the next we deal with the following smooth Gauss Weierstrass singular integral operators $W_{r, \xi}(f ; x)$ defined as follows.

For $r \in \mathbb{N}$ and $n \in \mathbb{Z}_{+}$we set $\alpha_{j}$ 's as in (2.1).

Let $f: \mathbb{R} \rightarrow \mathbb{R}$ be Lebesgue measurable, we define for $x \in \mathbb{R}, \xi>0$ the Lebesgue integral

$$
W_{r, \xi}(f ; x):=\frac{1}{\sqrt{\pi \xi}} \int_{-\infty}^{\infty}\left(\sum_{j=0}^{r} \alpha_{j} f(x+j t)\right) e^{-t^{2} / \xi} d t .
$$

We assume that $W_{r, \xi}(f ; x) \in \mathbb{R}$ for all $x \in \mathbb{R}$.

We mention the useful here formula

$$
\int_{0}^{\infty} t^{k} e^{-t^{2} / \xi} d t=\frac{1}{2} \Gamma\left(\frac{k+1}{2}\right) \xi^{\frac{k+1}{2}}, \text { for any } k>-1
$$

We also need to introduce $\delta_{k}$ 's as in $(2.4)$.

Proposition 36. Let $f \in C^{1}(\mathbb{R})$ be defined as above in this section, and assume that $W_{2, \xi}(f ; x) \in \mathbb{R}$ for all $x \in \mathbb{R}$. Then

$$
\left|W_{2, \xi}(f ; x)-f(x)\right| \leq \frac{2}{\sqrt{\pi \xi}} \int_{0}^{\infty}\left(\int_{0}^{|t|} \omega_{2}\left(f^{\prime}, w\right) d w\right) e^{-\frac{t^{2}}{\xi}} d t .
$$

Proof. In Theorem 1 of [3] we use $n=1, r=2$.

We present the Lipschitz type result corresponding to the Theorem 1 of [3].

Theorem 37. Let $f \in \mathrm{C}^{\mathfrak{n}}(\mathbb{R}), \mathrm{n} \in \mathbb{Z}^{+}$and assume that $W_{r, \xi}(f ; x) \in \mathbb{R}$ for all $x \in \mathbb{R}$. Furthermore we assume the following Lipschitz condition: $\omega_{\mathrm{r}}\left(\mathrm{f}^{(\mathfrak{n})}, \delta\right) \leq \mathrm{K} \delta^{\mathrm{r}-1+\gamma}, \mathrm{K}>0,0<\gamma \leq 1$, for any $\delta>0$. Then it holds that

$$
\begin{aligned}
& \leq W_{r, \xi}(f ; x)-f(x)-\sum_{m=1}^{\lfloor n / 2\rfloor} f^{(2 m)}(x) \delta_{2 m} \frac{1}{m !}\left(\frac{\xi}{4}\right)^{m} \mid \\
& \leq \frac{K}{\sqrt{\pi}} \frac{\Gamma(\gamma+r)}{\Gamma(n+\gamma+r)} \Gamma\left(\frac{n+r+\gamma}{2}\right) \xi^{\frac{n+r+\gamma-1}{2}} .
\end{aligned}
$$


In L.H.S.(4.4) the sum collapses when $\mathrm{n}=1$.

Proof. As in the proof of Theorem 1, of [3], we get again that

$$
W_{r, \xi}(f ; x)-f(x)=\sum_{k=1}^{n} \frac{f^{(k)}(x)}{k !} \delta_{k} \frac{1}{\sqrt{\pi \xi}}\left(\int_{-\infty}^{\infty} t^{k} e^{-\frac{t^{2}}{\xi}} d t\right)+\mathcal{R}_{n}^{*},
$$

where

$$
\mathcal{R}_{\mathrm{n}}^{*}:=\frac{1}{\sqrt{\pi \xi}} \int_{-\infty}^{\infty} \mathcal{R}_{\mathrm{n}}(0, \mathrm{t}) \mathrm{e}^{-\frac{\mathrm{t}^{2}}{\xi}} \mathrm{dt}
$$

with

$$
\mathcal{R}_{\mathrm{n}}(0, \mathrm{t}):=\int_{0}^{\mathrm{t}} \frac{(\mathrm{t}-w)^{\mathrm{n}-1}}{(\mathrm{n}-1) !} \tau(w) \mathrm{d} w
$$

and

$$
\tau(w):=\sum_{j=0}^{r} \alpha_{j} j^{n} f^{(n)}(x+j w)-\delta_{n} f^{(n)}(x) .
$$

Also we get

$$
\left|\mathcal{R}_{n}(0, t)\right| \leq \int_{0}^{|t|} \frac{(|t|-w)^{n-1}}{(n-1) !} \omega_{r}\left(f^{(n)}, w\right) d w .
$$

Using the Lipschitz type condition we obtain again

$$
\left|\mathcal{R}_{\mathrm{n}}(0, \mathrm{t})\right| \leq \frac{\mathrm{K}|\mathrm{t}|^{\mathrm{n}+\mathrm{r}+\gamma-1} \Gamma(\gamma+\mathrm{r})}{\Gamma(\mathrm{n}+\gamma+\mathrm{r})}
$$

and, using (4.2), we obtain

$$
\begin{aligned}
& \left|\mathcal{R}_{\mathrm{n}}^{*}\right| \leq \frac{1}{\sqrt{\pi \xi}} \int_{-\infty}^{\infty} \frac{\mathrm{K}|\mathrm{t}|^{\mathrm{n}+\mathrm{r}+\gamma-1} \Gamma(\gamma+\mathrm{r})}{\Gamma(\mathrm{n}+\gamma+\mathrm{r})} e^{-\frac{\mathrm{t}^{2}}{\xi}} \mathrm{dt} \\
& =\frac{\mathrm{K}}{\sqrt{\pi \xi}} \frac{\Gamma(\gamma+r)}{\Gamma(n+\gamma+r)} \int_{-\infty}^{\infty}|t|^{n+r+\gamma-1} e^{-\frac{t^{2}}{\xi}} d t \\
& =\frac{2 \mathrm{~K}}{\sqrt{\pi \xi}} \frac{\Gamma(\gamma+r)}{\Gamma(n+\gamma+r)} \int_{0}^{\infty} t^{n+r+\gamma-1} e^{-\frac{t^{2}}{\xi}} d t \\
& \stackrel{(4.2)}{=} \frac{\mathrm{K}}{\sqrt{\pi}} \frac{\Gamma(\gamma+r)}{\Gamma(n+\gamma+r)} \Gamma\left(\frac{n+r+\gamma}{2}\right) \xi^{\frac{n+r+\gamma-1}{2}} \text {. }
\end{aligned}
$$

We notice also that

$$
\begin{aligned}
& W_{r, \xi}(f ; x)-f(x)-\sum_{k=1}^{n} \frac{f^{(k)}(x)}{k !} \delta_{k} \frac{1}{\sqrt{\pi \xi}}\left(\int_{-\infty}^{\infty} t^{k} e^{-\frac{t^{2}}{\xi}} d t\right)= \\
& W_{r, \xi}(f ; x)-f(x)-\sum_{m=1}^{\left\lfloor\frac{n}{2}\right\rfloor}\left[\frac{f^{(2 m)}(x)}{(2 m) ! \sqrt{\pi}} \delta_{2 m} \Gamma\left(\frac{2 m+1}{2}\right) \xi^{m}\right]=\mathcal{R}_{n}^{*} .
\end{aligned}
$$


Furthermore we have that

$$
\begin{gathered}
\frac{1}{(2 m) ! \sqrt{\pi}} \Gamma\left(\frac{2 m+1}{2}\right)= \\
=\frac{1}{(2 m) \cdot(2 m-1) \cdot \ldots \cdot 3 \cdot 2 \cdot 1} \cdot \frac{1}{\sqrt{\pi}} \cdot \frac{2 m-1}{2} \cdot \frac{2 m-3}{2} \cdot \ldots \cdot \frac{3}{2} \cdot \frac{1}{2} \Gamma\left(\frac{1}{2}\right) \\
=\frac{1}{m !}\left(\frac{1}{4}\right)^{m} .
\end{gathered}
$$

By (4.10), (4.11) and (4.12) we complete the proof of the theorem.

Corollary 38. Let $f \in C^{1}(\mathbb{R})$, and assume that $W_{2, \xi}(f ; x) \in \mathbb{R}$ for all $x \in \mathbb{R}$. Furthermore we assume the following Lipschitz condition: $\omega_{2}\left(f^{\prime}, \delta\right) \leq \mathrm{K} \delta^{1+\gamma}, \mathrm{K}>0,0<\gamma \leq 1$, for any $\delta>0$. Then

$$
\left|W_{2, \xi}(f ; x)-f(x)\right| \leq \frac{K}{(\gamma+2) \sqrt{\pi}} \Gamma\left(\frac{3+\gamma}{2}\right) \xi^{\frac{2+\gamma}{2}} .
$$

Proof. In Theorem 37 we use $n=1, r=2$.

For the case $n=0$ we have

Theorem 39. Let $\mathrm{f}$ be defined as above in this section, with $\mathrm{n}=0$. Furthermore we assume the following Lipschitz condition: $\omega_{\mathrm{r}}(\mathrm{f}, \delta) \leq \mathrm{K} \delta^{\mathrm{r}-1+\gamma}, \mathrm{K}>0,0<\gamma \leq 1$, for any $\delta>0$. It holds that

$$
\left|W_{r, \xi}(f ; x)-f(x)\right| \leq \frac{K}{\sqrt{\pi}} \Gamma\left(\frac{r+\gamma}{2}\right) \xi^{\frac{r+\gamma-1}{2}} .
$$

Proof. As in the proof of Corollary 1, of [3], with $n=0$, using the Lipschitz type condition, we get that

$$
\begin{aligned}
\left|W_{r, \xi}(f ; x)-f(x)\right| & \leq \frac{2}{\sqrt{\pi \xi}} \int_{0}^{\infty} \omega_{r}(f, t) e^{-\frac{t^{2}}{\xi}} d t \\
& \leq \frac{2}{\sqrt{\pi \xi}} \int_{0}^{\infty} K t^{r-1+\gamma} e^{-\frac{t^{2}}{\xi}} d t \\
& \stackrel{(4.2)}{=} \frac{K}{\sqrt{\pi}} \Gamma\left(\frac{r+\gamma}{2}\right) \xi \frac{r+\gamma-1}{2} .
\end{aligned}
$$

This completes the proof of Theorem 39 .

Corollary 40. Let $\mathrm{f}$ be defined as above in this section, with $\mathrm{n}=0$. Furthermore we assume the following Lipschitz condition: $\omega_{2}(f, \delta) \leq \mathrm{K} \delta^{1+\gamma}, \mathrm{K}>0,0<\gamma \leq 1$, for any $\delta>0$. Then

$$
\left|W_{2, \xi}(f ; x)-f(x)\right| \leq \frac{K}{\sqrt{\pi}} \Gamma\left(\frac{2+\gamma}{2}\right) \xi^{\frac{\gamma+1}{2}} .
$$

Proof. In Theorem 39 we use $r=2$. 
In the next we consider $\mathrm{f} \in \mathrm{C}^{\mathrm{n}}(\mathbb{R}), \mathrm{n} \geq 2$ even and the simple smooth singular operator of symmetric convolution type

$$
W_{\xi}\left(f, x_{0}\right):=\frac{1}{\sqrt{\pi \xi}} \int_{-\infty}^{\infty} f\left(x_{0}+y\right) e^{-y^{2} / \xi} d y, \text { for all } x_{0} \in \mathbb{R}, \xi>0 .
$$

That is

$$
W_{\xi}\left(f ; x_{0}\right)=\frac{1}{\sqrt{\pi \xi}} \int_{0}^{\infty}\left(f\left(x_{0}+y\right)+f\left(x_{0}-y\right)\right) e^{-y^{2} / \xi} d y, \text { for all } x_{0} \in \mathbb{R}, \quad \xi>0 .
$$

We assume that $f$ is such that

$$
W_{\xi}\left(f ; x_{0}\right) \in \mathbb{R}, \quad \forall x_{0} \in \mathbb{R}, \forall \xi>0 \text { and } \omega_{2}\left(f^{(n)}, h\right)<\infty, h>0
$$

Note that $W_{1, \xi}=W_{\xi}$ and if $W_{\xi}\left(f ; x_{0}\right) \in \mathbb{R}$ then $W_{r, \xi}\left(f ; x_{0}\right) \in \mathbb{R}$.

Proposition 41. Assume $\mathrm{f} \in \mathrm{C}^{\mathrm{n}}(\mathbb{R}), \mathrm{\omega}_{2}(\mathrm{f}, \mathrm{h})<\infty, \mathrm{h}>0$. Furthermore we assume the following Lipschitz condition: $\omega_{2}(\mathrm{f}, \delta) \leq \mathrm{K} \delta^{1+\gamma}, \mathrm{K}>0,0<\gamma \leq 1$, for any $\delta>0$. Then

$$
\left\|W_{\xi}(f)-f\right\|_{\infty} \leq \frac{K}{2 \sqrt{\pi}} \Gamma\left(\frac{2+\gamma}{2}\right) \xi^{\frac{\gamma+1}{2}} .
$$

Proof. Using Proposition 1 of [3] we obtain

$$
\begin{aligned}
\left|W_{\xi}\left(f ; x_{0}\right)-f\left(x_{0}\right)\right| & \leq \frac{1}{\sqrt{\pi \xi}} \int_{0}^{\infty} \omega_{2}(f, y) e^{-y^{2} / \xi} d y \\
& \leq \frac{1}{\sqrt{\pi \xi}} \int_{0}^{\infty} K y^{1+\gamma} e^{-y^{2} / \xi} d y \\
& \stackrel{(4.2)}{=} \frac{K}{2 \sqrt{\pi}} \Gamma\left(\frac{2+\gamma}{2}\right) \xi^{\frac{\gamma+1}{2}},
\end{aligned}
$$

proving the claim of the proposition.

Define the quantity

$$
\bar{K}_{2}\left(x_{0}\right):=W_{\xi}\left(f ; x_{0}\right)-f\left(x_{0}\right)-\sum_{\rho=1}^{n / 2} f^{(2 \rho)}\left(x_{0}\right) \frac{1}{\rho !}\left(\frac{\xi}{4}\right)^{\rho} .
$$

We give

Theorem 42. Let $\mathrm{f} \in \mathrm{C}^{\mathrm{n}}(\mathbb{R}), \mathrm{n}$ even, $\mathrm{W}_{\xi}(\mathrm{f})$ real valued. Furthermore we assume the following Lipschitz condition: $\omega_{2}\left(f^{(n)}, \delta\right) \leq K \delta^{1+\gamma}, K>0,0<\gamma \leq 1$, for any $\delta>0$. Then

$$
\left|\overline{\mathrm{K}}_{2}\left(\mathrm{x}_{0}\right)\right| \leq \frac{\mathrm{K}}{\mathrm{n} ! 2 \sqrt{\pi}} \Gamma\left(\frac{\mathrm{n}+\gamma+2}{2}\right) \xi^{\frac{\mathrm{n}+\gamma+1}{2}} .
$$


Proof. Using Theorem 6 of [3] we obtain

$$
\begin{aligned}
\left|\overline{\mathrm{K}}_{2}\left(x_{0}\right)\right| & \leq \frac{1}{n ! \sqrt{\pi \xi}} \int_{0}^{\infty} \omega_{2}\left(f^{(n)}, y\right) y^{n} e^{-y^{2} / \xi} d y \\
& \leq \frac{1}{n ! \sqrt{\pi \xi}} \int_{0}^{\infty} K y^{1+\gamma} y^{n} e^{-y^{2} / \xi} d y \\
& \stackrel{(4.2)}{=} \frac{K}{n ! 2 \sqrt{\pi}} \Gamma\left(\frac{n+\gamma+2}{2}\right) \xi^{\frac{n+\gamma+1}{2}},
\end{aligned}
$$

proving the claim of the theorem.

In particular we have

Corollary 43. Let $\mathrm{f} \in \mathrm{C}^{4}(\mathbb{R})$ such that $\mathrm{W}_{\xi}(\mathrm{f})$ is real valued. Furthermore we assume the following Lipschitz condition: $\omega_{2}\left(f^{(4)}, \delta\right) \leq K \delta^{1+\gamma}, K>0,0<\gamma \leq 1$, for any $\delta>0$. Then

$$
\left|\overline{\mathrm{K}}_{2}\left(\mathrm{x}_{0}\right)\right| \leq \frac{\mathrm{K}}{48 \sqrt{\pi}} \Gamma\left(\frac{\gamma+6}{2}\right) \xi^{\frac{\gamma+5}{2}} .
$$

Proof. In Theorem 42 we use $n=4$.

We also give

Corollary 44. Let $\mathrm{f} \in \mathrm{C}^{2}(\mathbb{R})$, such that

$$
\omega_{2}\left(f^{\prime \prime},|y|\right) \leq 2 A|y|^{\gamma}, \quad 0<\gamma \leq 2, \quad A>0 .
$$

Then for $\mathrm{x}_{0} \in \mathbb{R}$ we have

$$
\left|W_{\xi}\left(f ; x_{0}\right)-f\left(x_{0}\right)-\frac{f^{\prime \prime}\left(x_{0}\right) \xi}{4}\right| \leq \frac{A}{(\gamma+1)(\gamma+2) \sqrt{\pi}} \Gamma\left(\frac{3+\gamma}{2}\right) \xi^{\frac{2+\gamma}{2}} .
$$

Inequality (4.25) is sharp, namely it is attained at $\mathrm{x}_{0}=0$ by

$$
f_{*}(y)=\frac{A|y|^{\gamma+2}}{(\gamma+1)(\gamma+2)} .
$$

Proof. In Theorem 7 of [3] we use $n=2$.

We also give

Corollary 45. Assume that $\omega_{2}(f, \xi)<\infty$ and $n=0$. Then

$$
\left\|W_{2, \xi}(f)-f\right\|_{\infty} \leq\left[\frac{2}{\sqrt{\pi}}+\frac{3}{2}\right] \omega_{2}(f, \sqrt{\xi}) .
$$


and as $\xi \rightarrow 0$,

$$
W_{2, \xi} \stackrel{\text { }}{\rightarrow} \text { I with rates. }
$$

Proof. By formula (37) of [3] with $r=2$.

Define the quantity

$$
\bar{K}_{1}:=\left\|W_{r, \xi}(f ; x)-f(x)-\sum_{m=1}^{\lfloor n / 2\rfloor} f^{(2 m)}(x) \delta_{2 m} \frac{1}{m !}\left(\frac{\xi}{4}\right)^{m}\right\|_{\infty, x} .
$$

We present

Corollary 46. Assuming $f \in \mathrm{C}^{2}(\mathbb{R})$ and $\omega_{2}\left(\mathrm{f}^{\prime \prime}, \xi\right)<\infty, \xi>0$ we have

$$
\begin{aligned}
\overline{\mathrm{K}}_{1} & =\left\|W_{2, \xi}(f ; x)-f(x)-f^{\prime \prime}(x) \delta_{2} \frac{\xi}{4}\right\|_{\infty, x} \\
& \leq\left\{\frac{1}{3 \sqrt{\pi}}+\frac{5}{16}\right\} \omega_{2}\left(f^{\prime \prime}, \sqrt{\xi}\right) \xi .
\end{aligned}
$$

That is as $\xi \rightarrow 0$ we get $\mathrm{W}_{2, \xi} \rightarrow \mathrm{I}$, pointwise with rates, given that $\left\|\mathrm{f}^{\prime \prime}\right\|_{\infty}<\infty$.

Proof. In Theorem 11 of [3] we use $r=n=2$.

We also present

Corollary 47. Assuming $f \in \mathrm{C}^{2}(\mathbb{R})$ and $\omega_{2}\left(f^{\prime \prime}, \xi\right)<\infty, \xi>0$ we have

$$
\begin{aligned}
\left\|\bar{K}_{2}(x)\right\|_{\infty, x} & =\left\|W_{\xi}\left(f ; x_{0}\right)-f\left(x_{0}\right)-f^{\prime \prime}\left(x_{0}\right) \frac{\xi}{4}\right\|_{\infty, x} \\
& \leq\left\{\frac{1}{6 \sqrt{\pi}}+\frac{5}{32}\right\} \omega_{2}\left(f^{\prime \prime}, \sqrt{\xi}\right) \xi .
\end{aligned}
$$

That is as $\xi \rightarrow 0$ we get $W_{\xi} \rightarrow \mathrm{I}$, pointwise with rates, given that $\left\|\mathrm{f}^{\prime \prime}\right\|_{\infty}<\infty$.

Proof. In Theorem 12 of [3] we use $n=2$.

\section{5. $\mathrm{L}_{\mathrm{p}}$ Convergence with Rates of Smooth Gauss Weierstrass Singular Integral Operators}

For $r \in \mathbb{N}$ and $n \in \mathbb{Z}_{+}$we let $\alpha_{j}$ as in (2.1). 
Let $f \in C^{\mathfrak{n}}(\mathbb{R})$ and $f^{(\mathfrak{n})} \in L_{p}(\mathbb{R}), 1 \leq p<\infty$, we define for $x \in \mathbb{R}, \xi>0$ the Lebesgue integral $W_{r, \xi}(f ; x)$ as in $(4.1)$.

The rth $L_{p}$-modulus of smoothness $\omega_{r}\left(f^{(n)}, h\right)_{p}$ was defined in (3.1). Here we have that $\omega_{\mathrm{r}}\left(\mathrm{f}^{(\mathrm{n})}, \mathrm{h}\right)_{\mathrm{p}}<\infty, \mathrm{h}>0$.

The $\delta_{k}$ 's were introduced in $(2.4)$.

We define

$$
\Delta(x):=W_{r, \xi}(f ; x)-f(x)-\sum_{m=1}^{\lfloor n / 2\rfloor} f^{(2 m)}(x) \delta_{2 m} \frac{1}{m !}\left(\frac{\xi}{4}\right)^{m} .
$$

We have the following results.

Corollary 48. Let $\mathrm{n} \in \mathbb{N}$ and the rest as above in this section. Then

$$
\|\Delta(x)\|_{2} \leq \frac{\sqrt{2 \tau} \xi^{\frac{n}{2}}}{(n-1) ! \sqrt[4]{\pi} \sqrt{(2 r+1)(2 n-1)}} \omega_{r}\left(f^{(n)}, \sqrt{\xi}\right)_{2},
$$

where

$$
0<\tau:=\left[\int_{0}^{\infty}(1+u)^{2 r+1} u^{2 n-1} e^{-u^{2}} d u-\int_{0}^{\infty} u^{2 n-1} e^{-u^{2}} d u\right]<\infty .
$$

Hence as $\xi \rightarrow 0$ we obtain $\|\Delta(\mathrm{x})\|_{2} \rightarrow 0$.

If additionally $f^{(2 m)} \in L_{2}(\mathbb{R}), m=1,2, \ldots,\left\lfloor\frac{n}{2}\right\rfloor$ then $\left\|W_{r, \xi}(f)-f\right\|_{2} \rightarrow 0$, as $\xi \rightarrow 0$.

Proof. In Theorem 1 of [4], we place $p=q=2$.

Corollary 49. Let $\mathrm{f}$ be as above in this section. In particular, for $\mathrm{n}=1$, we have

$$
\left\|W_{r, \xi}(f ; \cdot)-f\right\|_{2} \leq \frac{\sqrt{2 \tau}}{\sqrt[4]{\pi} \sqrt{(2 r+1)}} \sqrt{\xi} \omega_{r}\left(f^{\prime}, \sqrt{\xi}\right)_{2},
$$

where

$$
0<\tau:=\left[\int_{0}^{\infty}(1+u)^{2 r+1} u e^{-u^{2}} d u-\frac{1}{2}\right]<\infty .
$$

Hence as $\xi \rightarrow 0$ we obtain $\left\|\mathrm{W}_{\mathrm{r}, \xi}(\mathrm{f} ; \cdot)-\mathrm{f}\right\|_{2} \rightarrow 0$.

Proof. In Theorem 1 of [4], we place $p=q=2, n=1$.

Corollary 50. Let $\mathrm{f}$ be as above in this section and $\mathrm{n}=2$. Then

$$
\left\|W_{r, \xi}(f ; x)-f(x)-\frac{f^{\prime \prime}(x) \delta_{2}}{4} \xi\right\|_{2} \leq \frac{\sqrt{2 \tau}}{\sqrt[4]{\pi} \sqrt{3(2 r+1)}} \xi \omega_{r}\left(f^{\prime \prime}, \sqrt{\xi}\right)_{2},
$$


where

$$
0<\tau:=\left[\int_{0}^{\infty}(1+u)^{2 r+1} u^{3} e^{-u^{2}} d u-\frac{1}{2}\right]<\infty .
$$

Hence as $\xi \rightarrow 0$ we obtain $\|\Delta(\mathrm{x})\|_{2} \rightarrow 0$.

If additionally $f^{\prime \prime} \in L_{2}(\mathbb{R})$, then $\left\|W_{r, \xi}(f)-f\right\|_{2} \rightarrow 0$, as $\xi \rightarrow 0$.

Proof. In Theorem 1 of [4], we place $p=q=n=2$.

Next we present the Lipschitz type result corresponding to Theorem 1 of [4].

Theorem 51. Let $\mathrm{p}, \mathrm{q}>1$ such that $\frac{1}{\mathrm{p}}+\frac{1}{\mathrm{q}}=1, \mathrm{n} \in \mathbb{N}$, and the rest as above in this section. Furthermore we assume the following Lipschitz condition: $\omega_{\mathrm{r}}\left(\mathrm{f}^{(\mathrm{n})}, \delta\right)_{\mathrm{p}} \leq \mathrm{K} \delta^{\mathrm{r}-1+\gamma}, \mathrm{K}>0$, $0<\gamma \leq 1$, for any $\delta>0$. Then

$$
\|\Delta(x)\|_{p} \leq \frac{\left(\Gamma\left(\frac{p(r-1+\gamma+n)+1}{2}\right)\right)^{\frac{1}{p}} 2^{\frac{(r+\gamma+n)}{2}} K \xi \frac{(r-1+\gamma+n)}{2}}{\left[(n-1) ! p \frac{r-\frac{1}{q}+\gamma+n}{2} q^{\frac{1}{2 q}} \pi^{\frac{1}{2 p}}(q(n-1)+1)^{\frac{1}{q}}(p(r-1+\gamma)+1)^{\frac{1}{p}}\right]} .
$$

Hence as $\xi \rightarrow 0$ we obtain $\|\Delta(\mathrm{x})\|_{\mathrm{p}} \rightarrow 0$.

If additionally $f^{(2 m)} \in L_{p}(\mathbb{R}), m=1,2, \ldots,\left\lfloor\frac{n}{2}\right\rfloor$ then $\left\|W_{r, \xi}(f)-f\right\|_{p} \rightarrow 0$, as $\xi \rightarrow 0$.

Proof. As in the proof of Theorem 1, [4], we get again

$$
I:=\int_{-\infty}^{\infty}|\Delta(x)|^{p} d x \leq c_{1}\left(\int_{-\infty}^{\infty}\left(\int_{0}^{|t|} \omega_{r}\left(f^{(n)}, w\right)_{p}^{p} d w\right)|t|^{n p-1} e^{-\frac{p t^{2}}{2 \xi}} d t\right)
$$

where

$$
c_{1}:=\frac{2^{\frac{p-1}{2}}}{q^{\frac{p-1}{2}} \sqrt{\pi \xi}((n-1) !)^{p}(q(n-1)+1)^{p / q}} .
$$

Using the Lipschitz condition, we obtain

$$
\begin{aligned}
I & \leq c_{1}\left(\int_{-\infty}^{\infty}\left(\int_{0}^{|t|}\left(K w^{r-1+\gamma}\right)^{p} d w\right)|t|^{n p-1} e^{-\frac{p t^{2}}{2 \xi}} d t\right) \\
& =\frac{c_{1} K^{p}}{(p(r-1+\gamma)+1)}\left(\int_{-\infty}^{\infty}|t|^{p(r-1+\gamma+n)} e^{-\frac{p t^{2}}{2 \xi}} d t\right) \\
& =\frac{2 c_{1} K^{p}}{(p(r-1+\gamma)+1)}\left(\int_{0}^{\infty} t^{p(r-1+\gamma+n)} e^{-\frac{p t^{2}}{2 \xi}} d t\right) \\
& \stackrel{(4.2)}{=} \frac{c_{1} K^{p} \Gamma\left(\frac{p(r-1+\gamma+n)+1}{2}\right)}{(p(r-1+\gamma)+1)}\left(\frac{2}{p}\right)^{\frac{p(r-1+\gamma+n)+1}{2}} \xi \frac{p(r-1+\gamma+n)+1}{2} .
\end{aligned}
$$


Thus we obtain

$$
I \leq \frac{K^{p} 2^{\frac{p(r+\gamma+n)}{2}} \Gamma\left(\frac{p(r-1+\gamma+n)+1}{2}\right) \xi^{\frac{p(r-1+\gamma+n)}{2}}}{q^{\frac{p-1}{2}} \sqrt{\pi}((n-1) !)^{p}(q(n-1)+1)^{p / q}(p(r-1+\gamma)+1) p^{\frac{p(r-1+\gamma+n)+1}{2}}} .
$$

That is finishing the proof of the theorem.

In particular we have

Corollary 52. Let $\mathrm{f}$ such that the following Lipschitz condition holds: $\omega_{7}\left(\mathrm{f}^{(4)}, \delta\right)_{2} \leq \mathrm{K} \delta^{6+\gamma}$, $\mathrm{K}>0,0<\gamma \leq 1$, for any $\delta>0$, and the rest as above in this section. Then

$$
\|\Delta(x)\|_{2} \leq \frac{K}{6} \sqrt{\frac{\Gamma\left(\frac{2 \gamma+21}{2}\right)}{7 \sqrt{\pi}(2 \gamma+13)}} \xi^{\frac{(\gamma+10)}{2}} .
$$

Hence as $\xi \rightarrow 0$ we obtain $\|\Delta(\mathrm{x})\|_{2} \rightarrow 0$.

If additionally $f^{(2 m)} \in L_{2}(\mathbb{R}), m=1,2$, then $\left\|W_{7, \xi}(f)-f\right\|_{2} \rightarrow 0$, as $\xi \rightarrow 0$.

Proof. In Theorem 51 we place $p=q=2, n=4$, and $r=7$.

The counterpart of Theorem 51 follows, case of $p=1$.

Theorem 53. Let $\mathrm{f} \in \mathrm{C}^{\mathrm{n}}(\mathbb{R})$ and $\mathrm{f}^{(\mathrm{n})} \in \mathrm{L}_{1}(\mathbb{R}), \mathrm{n} \in \mathbb{N}$. Furthermore we assume the following Lipschitz condition: $\omega_{\mathrm{r}}\left(\mathrm{f}^{(\mathfrak{n})}, \delta\right)_{1} \leq \mathrm{K}^{\mathrm{r}-1+\gamma}, \mathrm{K}>0,0<\gamma \leq 1$, for any $\delta>0$. Then

$$
\|\Delta(x)\|_{1} \leq \frac{K}{(n-1) !(r+\gamma) \sqrt{\pi}} \Gamma\left(\frac{r+\gamma+n}{2}\right) \xi \frac{r+\gamma+n-1}{2} .
$$

Hence as $\xi \rightarrow 0$ we obtain $\|\Delta(\mathrm{x})\|_{1} \rightarrow 0$.

If additionally $f^{(2 m)} \in L_{1}(\mathbb{R}), m=1,2, \ldots,\left\lfloor\frac{\mathfrak{n}}{2}\right\rfloor$ then $\left\|W_{r, \xi}(f)-f\right\|_{1} \rightarrow 0$, as $\xi \rightarrow 0$.

Proof. As in the proof of Theorem 2, [4] we get

$$
\|\Delta(x)\|_{1} \leq \frac{1}{(n-1) ! \sqrt{\pi \xi}}\left(\int_{-\infty}^{\infty}\left(\int_{0}^{|t|} \omega_{r}\left(f^{(n)}, w\right)_{1} d w\right)|t|^{n-1} e^{-t^{2} / \xi} d t\right)
$$

Consequently we have 


$$
\begin{aligned}
\|\Delta(x)\|_{1} & \leq \frac{1}{(n-1) ! \sqrt{\pi \xi}}\left(\int_{-\infty}^{\infty}\left(\int_{0}^{|t|} K w^{r-1+\gamma} d w\right)|t|^{n-1} e^{-t^{2} / \xi} d t\right) \\
& =\frac{K}{(n-1) ! \sqrt{\pi \xi}}\left(\int_{-\infty}^{\infty}\left(\frac{|t|^{\mid r+\gamma}}{r+\gamma}\right)|t|^{n-1} e^{-t^{2} / \xi} d t\right) \\
& =\frac{K}{(n-1) !(r+\gamma) \sqrt{\pi \xi}}\left(\int_{-\infty}^{\infty}|t|^{r+\gamma+n-1} e^{-t^{2} / \xi} d t\right) \\
& =\frac{2 K}{(n-1) !(r+\gamma) \sqrt{\pi \xi}}\left(\int_{0}^{\infty} t^{r+\gamma+n-1} e^{-t^{2} / \xi} d t\right) \\
& \stackrel{(4.2)}{=} \frac{K}{(n-1) !(r+\gamma) \sqrt{\pi \xi}} \Gamma\left(\frac{r+\gamma+n}{2}\right) \xi^{\frac{r+\gamma+n}{2}} .
\end{aligned}
$$

We have gotten that

$$
\|\Delta(x)\|_{1} \leq \frac{K}{(n-1) !(r+\gamma) \sqrt{\pi}} \Gamma\left(\frac{r+\gamma+n}{2}\right) \xi \frac{r+\gamma+n-1}{2} .
$$

Hence the validity of (5.14).

Corollary 54. Let $\mathrm{f} \in \mathrm{C}^{2}(\mathbb{R})$ and $\mathrm{f}^{\prime \prime} \in \mathrm{L}_{1}(\mathbb{R})$. Furthermore we assume the following Lipschitz condition: $\omega_{2}\left(f^{\prime \prime}, \delta\right)_{1} \leq \mathrm{K} \delta^{1+\gamma}, \mathrm{K}>0,0<\gamma \leq 1$, for any $\delta>0$. Then

$$
\|\Delta(x)\|_{1} \leq \frac{K}{(2+\gamma) \sqrt{\pi}} \Gamma\left(\frac{4+\gamma}{2}\right) \xi^{\frac{\gamma+3}{2}} .
$$

Hence as $\xi \rightarrow 0$ we obtain $\|\Delta(\mathrm{x})\|_{1} \rightarrow 0$.

Also we get $\left\|W_{2, \xi}(f)-f\right\|_{1} \rightarrow 0$, as $\xi \rightarrow 0$.

Proof. In Theorem 53 we place $n=r=2$.

Next, when $n=0$ we get

Proposition 55. Let $\mathrm{r} \in \mathbb{N}$ and the rest as above. Then

$$
\left\|W_{r, \xi}(f)-f\right\|_{2} \leq \frac{2^{\frac{3}{4}} \theta^{\frac{1}{2}}}{q^{\frac{1}{4}} \pi^{\frac{1}{4}}} \omega_{r}(f, \sqrt{\xi})_{2},
$$

where

$$
0<\theta:=\int_{0}^{\infty}(1+t)^{2 r} e^{-t^{2}} d t<\infty
$$

Hence as $\xi \rightarrow 0$ we obtain $W_{r, \xi} \rightarrow$ unit operator $I$ in the $L_{2}$ norm, $p>1$.

Proof. In the proof of Proposition 1 of [4] we use $p=q=2$.

We continue with 
Proposition 56. Let $\mathrm{p}, \mathrm{q}>1$ such that $\frac{1}{\mathrm{p}}+\frac{1}{\mathrm{q}}=1$ and the rest as above. Furthermore we assume the following Lipschitz condition: $\omega_{\mathrm{r}}(\mathrm{f}, \delta)_{\mathrm{p}} \leq \mathrm{K} \delta^{\mathrm{r}-1+\gamma}, \mathrm{K}>0,0<\gamma \leq 1$, for any $\delta>0$. Then

$$
\left\|W_{r, \xi}(f)-f\right\|_{p} \leq \sqrt[p]{\Gamma\left(\frac{p(r-1+\gamma)+1}{2}\right)}\left(\frac{2}{p}\right)^{\frac{r+\gamma}{2}}\left(\frac{p}{q}\right)^{\frac{1}{2 q}} \frac{K}{\sqrt[p]{\pi}} \xi^{\frac{(r-1+\gamma)}{2}} .
$$

Hence as $\xi \rightarrow 0$ we obtain $W_{r, \xi} \rightarrow$ unit operator $I$ in the $L_{p}$ norm, $p>1$.

Proof. As in the proof of Proposition 1 of [4] we find

$$
\begin{gathered}
\int_{-\infty}^{\infty}\left|W_{r, \xi}(f ; x)-f(x)\right|^{p} d x \\
\leq \frac{2}{(\pi \xi)^{\frac{p}{2}}}\left(\frac{2 \pi \xi}{q}\right)^{\frac{p}{2 q}} \int_{0}^{\infty} \omega_{r}(f, t)_{p}^{p} e^{-\frac{p t^{2}}{2 \xi} d t} \\
\leq \frac{2 K^{p}}{(\pi \xi)^{\frac{p}{2}}}\left(\frac{2 \pi \xi}{q}\right)^{\frac{p}{2 q}} \int_{0}^{\infty} t^{p(r-1+\gamma)} e^{-\frac{p t^{2}}{2 \xi} d t} \\
\stackrel{(4.2)}{=} \frac{K^{p}}{\pi^{\frac{p}{2}}}\left(\frac{2 \pi}{q}\right)^{\frac{p}{2 q}}\left(\frac{2}{p}\right)^{\frac{p(r-1+\gamma)+1}{2}} \Gamma\left(\frac{p(r-1+\gamma)+1}{2}\right) \xi \frac{p(r-1+\gamma)}{2} .
\end{gathered}
$$

We have established the claim of the proposition.

Corollary 57. Let $f$ such that the following Lipschitz condition holds: $\omega_{4}(f, \delta)_{2} \leq K \delta^{3+\gamma}$, $\mathrm{K}>0,0<\gamma \leq 1$, for any $\delta>0$, and the rest as above in this section. Then

$$
\left\|W_{4, \xi}(f)-f\right\|_{2} \leq \sqrt{\Gamma\left(\frac{2 \gamma+7}{2}\right)} \frac{K}{\sqrt{\pi}} \xi^{\frac{(3+\gamma)}{2}} .
$$

Hence as $\xi \rightarrow 0$ we obtain $W_{4, \xi} \rightarrow$ unit operator $I$ in the $\mathrm{L}_{2}$ norm.

Proof. In Proposition 56 we place $p=q=2$ and $r=4$.

In the $\mathrm{L}_{1}$ case, $\mathrm{n}=0$ we have

Proposition 58. It holds

$$
\left\|W_{2, \xi} f-f\right\|_{1} \leq\left(\frac{2}{\sqrt{\pi}}+\frac{3}{2}\right) \omega_{2}(f, \sqrt{\xi})_{1} .
$$

Hence as $\xi \rightarrow 0$ we get $\mathrm{W}_{2, \xi} \rightarrow \mathrm{I}$ in the $\mathrm{L}_{1}$ norm.

Proof. In the proof of Proposition 2 of [4] we use $r=2$.

Proposition 59. We assume the following Lipschitz condition: $\omega_{\mathrm{r}}(\mathrm{f}, \delta)_{1} \leq \mathrm{K} \delta^{\mathrm{r}-1+\gamma}, \mathrm{K}>0$, $0<\gamma \leq 1$, for any $\delta>0$. Then

$$
\left\|W_{r, \xi} f-f\right\|_{1} \leq \frac{K}{\sqrt{\pi}} \Gamma\left(\frac{r+\gamma}{2}\right) \xi^{\frac{r-1+\gamma}{2}} .
$$


Hence as $\xi \rightarrow 0$ we get $\mathrm{W}_{\mathrm{r}, \xi} \rightarrow \mathrm{I}$ in the $\mathrm{L}_{1}$ norm.

Proof. As in the proof of Proposition 2 of [4] we get

$$
\begin{aligned}
\int_{-\infty}^{\infty}\left|W_{r, \xi}(f ; x)-f(x)\right| d x & \leq \frac{1}{\sqrt{\pi \xi}} \int_{-\infty}^{\infty} \omega_{r}(f,|t|)_{1} e^{-t^{2} / \xi} d t \\
& \leq \frac{1}{\sqrt{\pi \xi}} \int_{-\infty}^{\infty} K|t|^{r-1+\gamma} e^{-t^{2} / \xi} d t \\
& =\frac{2 K}{\sqrt{\pi \xi}} \int_{0}^{\infty} t^{r-1+\gamma} e^{-t^{2} / \xi} d t \\
& \stackrel{(4.2)}{=} \frac{K}{\sqrt{\pi}} \Gamma\left(\frac{r+\gamma}{2}\right) \xi^{\frac{r-1+\gamma}{2}} .
\end{aligned}
$$

We have proved the claim of the proposition.

Corollary 60. Assume the following Lipschitz condition: $\omega_{2}(f, \delta)_{1} \leq \mathrm{K} \delta^{1+\gamma}, \mathrm{K}>0,0<\gamma \leq$ 1 , for any $\delta>0$. Then

$$
\left\|W_{2, \xi} f-f\right\|_{1} \leq \frac{K}{\sqrt{\pi}} \Gamma\left(\frac{2+\gamma}{2}\right) \xi^{\frac{1+\gamma}{2}} .
$$

Hence as $\xi \rightarrow 0$ we get $\mathrm{W}_{2, \xi} \rightarrow \mathrm{I}$ in the $\mathrm{L}_{1}$ norm.

Proof. In Proposition 59 we place $r=2$.

In the next we consider $f \in C^{n}(\mathbb{R})$ and $f^{(n)} \in L_{p}(\mathbb{R}), n=0$ or $n \geq 2$ even, $1 \leq p<\infty$ and the similar smooth singular operator of symmetric convolution type

$$
W_{\xi}(f ; x)=\frac{1}{\sqrt{\pi \xi}} \int_{-\infty}^{\infty} f(x+y) e^{-y^{2} / \xi} d y, \text { for all } x \in \mathbb{R}, \xi>0 .
$$

Denote

$$
K(x):=W_{\xi}(f ; x)-f(x)-\sum_{\rho=1}^{n / 2} \frac{f^{(2 \rho)}(x)}{\rho !} \cdot\left(\frac{\xi}{4}\right)^{\rho}
$$

We give

Theorem 61. Let $\mathrm{n} \geq 2$ even and the rest as above. Then

$$
\|K(x)\|_{2} \leq \sqrt{\frac{\tilde{\tau}}{10 \sqrt{\pi}(2 n-1)}} \frac{\xi^{\frac{n}{2}}}{(n-1) !} \omega_{2}\left(f^{(n)}, \sqrt{\xi}\right)_{2},
$$

where

$$
0<\tilde{\tau}=\int_{0}^{\infty}\left((1+u)^{5}-1\right) u^{2 n-1} e^{-u^{2}} d u<\infty .
$$

Hence as $\xi \rightarrow 0$ we get $\|\mathrm{K}(\mathrm{x})\|_{2} \rightarrow 0$. 
If additionally $\mathrm{f}^{(2 \mathrm{~m})} \in \mathrm{L}_{2}(\mathbb{R}), \mathrm{m}=1,2, \ldots, \frac{\mathfrak{n}}{2}$ then $\left\|W_{\xi}(f)-f\right\|_{2} \rightarrow 0$, as $\xi \rightarrow 0$.

Proof. In the proof of Theorem 3 of [4] we use $p=q=2$.

It follows a Lipschitz type approximation result.

Theorem 62. Let $\mathrm{p}, \mathrm{q}>1$ such that $\frac{1}{\mathrm{p}}+\frac{1}{\mathrm{q}}=1, \mathrm{n} \geq 2$ even and the rest as above. Furthermore we assume the following Lipschitz condition: $\omega_{2}\left(f^{(n)}, \delta\right)_{p} \leq K \delta^{\gamma+1}, K>0,0<\gamma \leq 1$, for any $\delta>0$. Then

$$
\|K(x)\|_{p} \leq \frac{K\left[\Gamma\left(\frac{p(\gamma+n+1)+1}{2}\right)\right]^{\frac{1}{p}}}{\sqrt{2} \pi^{\frac{1}{2 p}}(n-1) ! p^{\frac{1}{2 p}} q^{\frac{1}{2 q}}[q(n-1)+1]^{\frac{1}{q}}[p(\gamma+1)+1]^{\frac{1}{p}}}\left(\frac{2}{p}\right)^{\frac{(\gamma+n+1)}{2}} \xi^{\frac{(\gamma+n+1)}{2}} .
$$

Hence as $\xi \rightarrow 0$ we get $\|\mathrm{K}(\mathrm{x})\|_{\mathrm{p}} \rightarrow 0$.

If additionally $\mathrm{f}^{(2 \mathrm{~m})} \in \mathrm{L}_{\mathfrak{p}}(\mathbb{R}), \mathrm{m}=1,2, \ldots, \frac{\mathfrak{n}}{2}$ then $\left\|W_{\xi}(f)-f\right\|_{p} \rightarrow 0$, as $\xi \rightarrow 0$.

Proof. As in the proof of Theorem 3, of [4] we find

$$
\begin{aligned}
& \int_{-\infty}^{\infty}|K(x)|^{p} d x \leq c_{2}\left(\int_{0}^{\infty}\left(\int_{0}^{y} \omega_{2}\left(f^{(n)}, t\right)_{p}^{p} d t\right) y^{p n-1} e^{-\frac{p y^{2}}{2 \xi}} d y\right) \\
& \leq K^{p} c_{2}\left(\int_{0}^{\infty}\left(\frac{y^{p(\gamma+1)+1}}{p(\gamma+1)+1}\right) y^{p n-1} e^{-\frac{p y^{2}}{2 \xi}} d y\right) \\
& =\frac{\mathrm{K}^{\mathrm{p}} \mathrm{c}_{2}}{\mathrm{p}(\gamma+1)+1}\left(\int_{0}^{\infty} \mathrm{y}^{\mathrm{p}(\gamma+\mathrm{n}+1)} e^{-\frac{\mathrm{p} y^{2}}{2 \varepsilon}} \mathrm{dy}\right) \\
& \stackrel{(4.2)}{=} \frac{\mathrm{K}^{\mathrm{p}} \mathrm{c}_{2}}{\mathrm{p}(\gamma+1)+1}\left(\frac{2}{\mathrm{p}}\right)^{\frac{\mathrm{p}(\gamma+n+1)+1}{2}} \\
& \cdot \frac{1}{2} \Gamma\left(\frac{p(\gamma+n+1)+1}{2}\right) \xi \frac{p(\gamma+n+1)+1}{2} \text {. }
\end{aligned}
$$

where here we denoted

$$
c_{2}:=\frac{1}{2^{\frac{p}{2 q}} q^{\frac{p}{2 q}}(q(n-1)+1)^{p / q}((n-1) !)^{p} \sqrt{\pi \xi}} .
$$

We have established the claim of the theorem.

Corollary 63. Assume the following Lipschitz condition: $\omega_{2}\left(f^{\prime \prime}, \delta\right)_{2} \leq \mathrm{K} \delta^{\gamma+1}, \mathrm{~K}>0,0<$ $\gamma \leq 1$, for any $\delta>0$, and the rest as above in this section. Then

$$
\|\mathrm{K}(\mathrm{x})\|_{2} \leq \sqrt{\frac{\left[\Gamma\left(\frac{2 \gamma+7}{2}\right)\right]}{\sqrt{\pi}[6 \gamma+9]}} \frac{K}{2} \xi \frac{(\gamma+3)}{2} .
$$

Hence as $\xi \rightarrow 0$ we get $\|\mathrm{K}(\mathrm{x})\|_{2} \rightarrow 0$.

If additionally $f^{\prime \prime} \in L_{2}(\mathbb{R})$, then $\left\|W_{\xi}(f)-f\right\|_{2} \rightarrow 0$, as $\xi \rightarrow 0$. 
Proof. In Theorem 62 we place $p=q=n=2$.

Theorem 64. Let $f \in C^{2}(\mathbb{R})$ and $f^{\prime \prime} \in L_{1}(\mathbb{R})$. Here $\mathrm{K}(\mathrm{x})=\mathrm{W}_{\xi}(f ; x)-f(x)-\frac{f^{\prime \prime}(x)}{4} \xi$. Then

$$
\|\mathrm{K}(x)\|_{1} \leq\left(\frac{1}{2 \sqrt{\pi}}+\frac{3}{8}\right) \omega_{2}\left(f^{\prime \prime}, \sqrt{\xi}\right)_{1} \xi .
$$

Hence as $\xi \rightarrow 0$ we obtain $\|\mathrm{K}(\mathrm{x})\|_{1} \rightarrow 0$.

Also $\left\|W_{\xi}(f)-f\right\|_{1} \rightarrow 0$, as $\xi \rightarrow 0$.

Proof. In the proof of Theorem 4 of [4] we use $n=2$.

The Lipschitz case of $p=1$ follows.

Theorem 65. Let $\mathrm{f} \in \mathrm{C}^{\mathrm{n}}(\mathbb{R})$ and $\mathrm{f}^{(\mathrm{n})} \in \mathrm{L}_{1}(\mathbb{R}), \mathrm{n} \geq 2$ even. Furthermore we assume the following Lipschitz condition: $\omega_{2}\left(\mathrm{f}^{(\mathrm{n})}, \delta\right)_{1} \leq \mathrm{K} \delta^{\gamma+1}, \mathrm{~K}>0,0<\gamma \leq 1$, for any $\delta>0$. Then

$$
\|K(x)\|_{1} \leq \frac{\Gamma\left(\frac{\gamma+n+2}{2}\right) K}{(n-1) !(\gamma+2) 2 \sqrt{\pi}} \xi^{\frac{\gamma+n+1}{2}} .
$$

Hence as $\xi \rightarrow 0$ we obtain $\|\mathrm{K}(\mathrm{x})\|_{1} \rightarrow 0$.

If additionally $f^{(2 m)} \in L_{1}(\mathbb{R}), m=1,2, \ldots, \frac{n}{2}$ then $\left\|W_{\xi}(f)-f\right\|_{1} \rightarrow 0$, as $\xi \rightarrow 0$.

Proof. As in the proof of Theorem 4 of [4] we have

$$
\begin{aligned}
\|K(x)\|_{1} & \leq \frac{1}{\sqrt{\pi \xi}} \int_{0}^{\infty}\left(\left(\int_{0}^{y} \omega_{2}\left(f^{(n)}, t\right)_{1} d t\right) \frac{y^{n-1}}{(n-1) !} e^{-y^{2} / \xi}\right) d y \\
& \leq \frac{1}{\sqrt{\pi \xi}} \int_{0}^{\infty}\left(\left(\int_{0}^{y} K t^{\gamma+1} d t\right) \frac{y^{n-1}}{(n-1) !} e^{-y^{2} / \xi}\right) d y \\
& =\frac{K}{(n-1) !(\gamma+2) \sqrt{\pi \xi}} \int_{0}^{\infty}\left(y^{\gamma+n+1} e^{-y^{2} / \xi}\right) d y \\
& \stackrel{(4.2)}{=} \frac{\Gamma\left(\frac{\gamma+n+2}{2}\right) K}{(n-1) !(\gamma+2) 2 \sqrt{\pi}} \xi^{\frac{\gamma+n+1}{2}} .
\end{aligned}
$$

We have proved the claim of the theorem.

Corollary 66. Let $f \in \mathrm{C}^{6}(\mathbb{R})$ and $\mathrm{f}^{(6)} \in \mathrm{L}_{1}(\mathbb{R})$. Furthermore we assume the following Lipschitz condition: $\omega_{2}\left(\mathrm{f}^{(6)}, \delta\right)_{1} \leq \mathrm{K} \delta^{\gamma+1}, \mathrm{~K}>0,0<\gamma \leq 1$, for any $\delta>0$. Then

$$
\|\mathrm{K}(x)\|_{1} \leq \frac{\Gamma\left(\frac{\gamma+8}{2}\right) \mathrm{K}}{240(\gamma+2) \sqrt{\pi}} \xi^{\frac{\gamma+7}{2}} .
$$

Hence as $\xi \rightarrow 0$ we obtain $\|\mathrm{K}(\mathrm{x})\|_{1} \rightarrow 0$.

If additionally $f^{(2 m)} \in L_{1}(\mathbb{R}), m=1,2,3$ then $\left\|W_{\xi}(f)-f\right\|_{1} \rightarrow 0$, as $\xi \rightarrow 0$. 
Proof. In Theorem 65 we place $n=6$.

The case of $n=0$ follows.

Proposition 67. Let $f$ as above in this section. Then

$$
\left\|W_{\xi}(f)-f\right\|_{2} \leq \sqrt{\frac{2}{\sqrt{\pi}}+\frac{19}{16}} \omega_{2}(f, \sqrt{\xi})_{2} .
$$

Hence as $\xi \rightarrow 0$ we obtain $\mathrm{W}_{\xi} \rightarrow \mathrm{I}$ in the $\mathrm{L}_{2}$ norm.

Proof. In the proof of Proposition 3 of [4] we use $p=q=2$.

The related Lipschitz case for $n=0$ comes next.

Proposition 68. Let $\mathrm{p}, \mathrm{q}>1$ such that $\frac{1}{\mathrm{p}}+\frac{1}{\mathrm{q}}=1$ and the rest as above. Furthermore we assume the following Lipschitz condition: $\omega_{2}(\mathrm{f}, \delta)_{\mathrm{p}} \leq \mathrm{K} \delta^{1+\gamma}, \mathrm{K}>0,0<\gamma \leq 1$, for any $\delta>0$. Then

$$
\left\|W_{\xi}(f)-f\right\|_{p} \leq\left(\frac{2}{p}\right)^{\frac{(1+\gamma)}{2}} \frac{\left[\Gamma\left(\frac{(1+\gamma) p+1}{2}\right)\right]^{\frac{1}{p}} K}{\pi^{\frac{1}{2 p}} p^{\frac{1}{2 p}} q^{\frac{1}{2 q}} \sqrt{2}} \xi^{\frac{(1+\gamma)}{2}} .
$$

Hence as $\xi \rightarrow 0$ we obtain $\mathrm{W}_{\xi} \rightarrow \mathrm{I}$ in the $\mathrm{L}_{\mathrm{p}}$ norm, $\mathrm{p}>1$.

Proof. As in the proof of Proposition 3 of [4] we get

$$
\begin{aligned}
& \int_{-\infty}^{\infty}\left|W_{\xi}(f ; x)-f(x)\right|^{p} d x \leq \frac{1}{\sqrt{\pi \xi}(2 q)^{\frac{p}{2 q}}} \int_{0}^{\infty} \omega_{2}(f, y)_{p}^{p} e^{\frac{-p y^{2}}{2 \xi}} d y \\
& \leq \frac{1}{\sqrt{\pi \xi}(2 q)^{\frac{p}{2 q}}} \int_{0}^{\infty}\left(K y^{1+\gamma}\right)^{p} e^{\frac{-p y^{2}}{2 \xi}} d y \\
& \stackrel{(4.2)}{=} \frac{K^{p}}{\sqrt{\pi}(2 q)^{\frac{p}{2 q}}}\left(\frac{2}{p}\right)^{\frac{(1+\gamma) p+1}{2}} \frac{1}{2} \Gamma\left(\frac{(1+\gamma) p+1}{2}\right) \xi^{\frac{(1+\gamma) p}{2}} .
\end{aligned}
$$

The proof of the claim is now completed.

A particular example follows

Corollary 69. Let $\mathrm{f}$ as above in this section. Furthermore we assume the following Lipschitz condition: $\omega_{2}(f, \delta)_{2} \leq \mathrm{K} \delta^{1+\gamma}, \mathrm{K}>0,0<\gamma \leq 1$, for any $\delta>0$. Then

$$
\left\|W_{\xi}(f)-f\right\|_{2} \leq \frac{K}{2} \sqrt{\frac{\Gamma\left(\frac{3+2 \gamma}{2}\right)}{\sqrt{\pi}}} \xi \frac{(1+\gamma)}{2} .
$$

Hence as $\xi \rightarrow 0$ we obtain $\mathrm{W}_{\xi} \rightarrow \mathrm{I}$ in the $\mathrm{L}_{2}$ norm.

Proof. In Proposition 68 we place $p=q=2$. 
We finish with the Lipschitz type result

Proposition 70. Assume the following Lipschitz condition: $\omega_{2}(\mathrm{f}, \delta)_{1} \leq \mathrm{K} \delta^{\gamma+1}, \mathrm{~K}>0$, $0<\gamma \leq 1$, for any $\delta>0$. It holds,

$$
\left\|W_{\xi} f-f\right\|_{1} \leq \frac{K}{2 \sqrt{\pi}} \Gamma\left(\frac{\gamma+2}{2}\right) \xi^{\frac{\gamma+1}{2}} .
$$

Hence as $\xi \rightarrow 0$ we get $\mathrm{W}_{\xi} \rightarrow \mathrm{I}$ in the $\mathrm{L}_{1}$ norm.

Proof. As in the proof of Proposition 4 of [4] we derive

$$
\begin{aligned}
\int_{-\infty}^{\infty}\left|W_{\xi}(f ; x)-f(x)\right| d x & \leq \frac{1}{\sqrt{\pi \xi}} \int_{0}^{\infty} \omega_{2}(f, y){ }_{1} e^{-y^{2} / \xi} d y \\
& \leq \frac{1}{\sqrt{\pi \xi}} \int_{0}^{\infty} K y^{\gamma+1} e^{-y^{2} / \xi} d y \\
& \stackrel{(4.2)}{=} \frac{K}{2 \sqrt{\pi}} \Gamma\left(\frac{\gamma+2}{2}\right) \xi^{\frac{\gamma+1}{2}} .
\end{aligned}
$$

We have established the claim.

Received: September 2009. Revised: July 2010.

\section{References}

[1] George A. Anastassiou, "Basic Convergence with Rates of Smooth Picard Singular Operators", J. Comput. Anal. Appl., 8 (2006), 313-334.

[2] George A. Anastassiou, "Lp convergence with rates of smooth Picard singular operators", Differential \& difference equations and applications, Hindawi Publ. Corp., New York, (2006), 31-45.

[3] George A. Anastassiou, Razvan A. Mezei, "Uniform Convergence with Rates of Smooth Gauss-Weierstrass Singular Integral Operators", Applicable Analysis, 88:7 (2009), 1015 — 1037.

[4] George A. Anastassiou, Razvan A. Mezei, "Lp Convergence with Rates of Smooth GaussWeierstrass Singular Operators", Nonlinear Studies, accepted 2009. 